\title{
Time evolution of entanglement entropy of moving mirrors influenced by strongly coupled quantum critical fields
}

\author{
Da-Shin Lee and Chen-Pin Yeh \\ Department of Physics, National Dong-Hwa University, \\ Hualien, Taiwan, R.O.C. \\ E-mail: dslee@mail.ndhu.edu.tw, chenpinyeh@mail.ndhu.edu.tw
}

ABSTRACT: The evolution of the Von Neumann entanglement entropy of a $n$-dimensional mirror influenced by the strongly coupled $d$-dimensional quantum critical fields with a dynamic exponent $z$ is studied by the holographic approach. The dual description is a $n+1$-dimensional probe brane moving in the $d+1$-dimensional asymptotic Lifshitz geometry ended at $r=r_{b}$, which plays a role as the UV energy cutoff. Using the holographic influence functional method, we find that in the linear response region, by introducing a harmonic trap for the mirror, which serves as a IR energy cutoff, the Von Neumann entropy at late times will saturate by a power-law in time for generic values of $z$ and $n$. The saturated value and the relaxation rate depend on the parameter $\alpha \equiv 1+(n+2) / z$, which is restricted to $1<\alpha<3$ but $\alpha \neq 2$. We find that the saturated values of the entropy are qualitatively different for the theories with $1<\alpha<2$ and $2<\alpha<3$. Additionally, the power law relaxation follows the rate $\propto t^{-2 \alpha-1}$. This probe brane approach provides an alternative way to study the time evolution of the entanglement entropy in the linear response region that shows the similar power-law relaxation behavior as in the studies of entanglement entropies based on Ryu-Takayanagi conjecture. We also compare our results with quantum Brownian motion in a bath of relativistic free fields.

KEYWORDS: Gauge-gravity correspondence, Holography and condensed matter physics (AdS/CMT), Quantum Dissipative Systems

ARXiv EPrint: 1904.06831 


\section{Contents}

1 Introduction 1

2 Nonequilibrium effective action and influence functional 4

3 The holography model and holographic influence functional $\quad 6$

4 Dynamics of a moving mirror $\quad 7$

$\begin{array}{lll}\text { 4.1 The pole contributions } & 12\end{array}$

4.1.1 The damping term dominated region $(\Delta \gg 1) \quad 12$

4.1.2 The kinetic term dominated region $(\Delta \ll 1) \quad 13$

$\begin{array}{lll}4.2 & \text { The cut contributions } & 13\end{array}$

5 Quantum uncertainties on the mirror and the entanglement entropy 14

6 Comparison with the environment of relativistic free field theory $\quad 20$

$\begin{array}{lll}7 & \text { Summary and outlook } & 22\end{array}$

8 Brief summary of the holographic influence functional method 24

\section{Introduction}

Entanglement entropies provide useful probes to non-local properties of quantum systems, which are important for understanding quantum phase transitions [1], and moreover are the key quantities in quantum information processing [2]. The idea of entanglement entropies has also received much attention in connection to the information paradox in black hole physics [3]. It is generally impossible to isolate a particular quantum system in which we are interested from its surrounding. Considering a full theory that describes the interaction between system and environment, from which all information like correlation functions can in principle be obtained. Tracing or integrating out the degrees of freedom of the environment to obtain an effective field theory for the system leads to a loss of information. If the quantum state in the full theory is a pure state, namely a zero entropy state, tracing out the environmental degrees of freedom yields a reduced density matrix for the degrees of freedom of the system, which typically becomes a mixed state with non-vanishing entropy. The Von-Neumann entropy is a measure of the loss of information in the process of integrating out some degrees of freedom in the full unitary system $[4,5]$. The effective theory can be described by the reduced density matrix $\rho_{r}$, which is obtained by tracing out the environmental variables in the full density matrix using the method of Feyman-Vernon influence functional [6]. The Von Neumann entropy is then defined by $-\operatorname{Tr} \rho_{r} \ln \rho_{r}$ with the 
trace over system's variables. In general, the effective theory for the system obtained in this way is not unitary, and it can lead to the dissipative and stochastic behavior of the system. This concept has been used in pioneering works on quantum Brownian motion [7-9] and general open quantum systems [4]. In particular, the relaxation of the system into equilibrium with the environment can be characterized by the time evolution of the entanglement entropy. The method of Feynman-Vernon influence functional has also been extended to the quantum field theory [7-11]. However, the influence functional can only be exactly derived if the environmental theories are Gaussian and their coupling with the system is linear $[4,12]$. In this paper, we would like to study the entanglement between a particle or a mirror and some strongly coupled quantum fields by a holographic construction of the influence functional that has been proposed in [13] and [14].

The idea of holographic duality is originally proposed as the correspondence between 4-dimensional conformal field theory and gravity theory in 5-dimensional anti-de Sitter space [15-17], and soon is generalized to other backgrounds and field theories. One aim is to provide a framework to study the strong coupling problems in the condensed matter systems (see [18] for a review). The holographic approach has also be adapted to tackle the problems of the Brownian motion of a particle moving in a strongly coupled environment $[13,14,19-34]$. The idea is that a particle immersed in a environment given by the quantum field corresponds to a bulk fundamental string ended at the boundary of the dual gravity theory. In the black hole background, the string undergoes random motion due to the Hawking radiation of the transverse fluctuation modes. This is the bulk dual of the thermal Brownian motion. A review on the holographic Brownian motion can be found in $[25,26]$. Another aim of the holographic duality is to understand the quantum behavior of black hole or quantum gravity in general. A recent proposal by Ryu and Takayanagi [35], that the entanglement entropy in the boundary theory is related to the minimal area in the gravity theory, has arisen the hope that quantum gravity may be formulated in the language of quantum information. Time evolution of the entanglement entropy has also been studied in this framework [36-38], as a measure of the relaxation rate for non-equilibrium systems and also as a probe to the black hole interior.

In this work, we apply a bottom-up holographic method, proposed in our earlier works [29] and [14], to study the evolution of the entanglement entropy for the system of a $n$-dimensional mirror in the environment of $d$-dimensional quantum critical theories with dynamical exponent $z$ at zero temperature. The holographic dual for such quantum critical theories has been proposed in [39] where the gravity theory is in the Lifshitz background (See [27, 28] for details). Several physical phenomena have been studied in this theory, including linear DC conductivity, power-law AC conductivity, and strange fermion behaviors [28, 40-43]. In our set-up, the bulk counterpart of the mirror is a $(n+1)$-brane in the Lifshitz geometry in $d+1$ dimensions. The dynamics of the mirror can be realized from the motion of the brane ended at the boundary of the bulk at the radius distance $r_{b}$. As explained in [29] and will also be reviewed in the appendix, this holographic identification is based upon the fact that the coupling of the brane to the boundary field shares similar feature as the coupling between the mirror and the environment quantum field, where the mirror of perfect reflection effectively sets the vanishing boundary condition for the 
field $[29,44]$. In the case of $n=0$, it becomes a fundamental string in the bulk and its end point describes the position of a particle in the boundary. Using the method of holographic influence functional, developed in [14], we are able to derive the reduced density matrix of the mirror for a given initial state. The trick to adopt is that in the linear response approximation, with an initial Gaussian state of the system, the reduced density matrix remains Gaussian when turning on the bilinear couplings between the system and environment, and is completely determined by the expectation values of the position operator $\hat{q}$, momentum operator $\hat{p}$ and their product $\hat{q} \hat{p}+\hat{p} \hat{q}$. Thus the time dependent reduced density matrix can be constructed from the expectation values with the influence functional, rather than solving the master equation for the reduced density matrix. The evolution of the entanglement entropy shows how the system equilibrate with the environment and lose the information about its initial state, as seen from the increase of the entropy comparing to the entropy in the initial state of the system.

To summarize, in this paper we consider quantum critical fields with $1<\alpha<3$ and $\alpha \neq 2$ where $\alpha \equiv 1+\frac{n+2}{z}$ that couple to the system of the mirror. Our results show that the Von Neumann entanglement entropy of the system relaxes by the power law in time as $t^{-2 \alpha-1}$ toward saturation. Since the boundary field has the dispersion relation $E \propto k^{z}$, in the $d-1$ spatial dimension the density of state $\rho(E) \propto E^{-1+\frac{d-1}{z}}$. For a $n$-dimensional mirror under consideration, the effective spatial dimension probed by the mirror is $n$. Thus, the environmental density of states available to the mirror would be $\rho(E) \propto E^{-1+\frac{n}{z}}$. Accordingly, the density of modes decreases as increasing $z$ or decreasing $n$. If the relaxation of the system is attributed to dissipation of energy of its degrees of freedom to the modes of the environment, the relaxation rate decreases as $\alpha$ is decreased. Moreover, we find that the saturate value of the entanglement entropy, $S(t \rightarrow \infty) \simeq\left(1-\frac{1}{\alpha}\right) \ln \left(\frac{r_{b}^{z}}{\Omega}\right)$ for $1<\alpha<2$ and $S(t \rightarrow \infty) \simeq\left(\frac{3}{2}-\frac{\alpha}{2}\right) \ln \left(\frac{r_{b}^{z}}{\Omega}\right)$ for $2<\alpha<3$, where $\Omega$ is the oscillation frequency of the harmonic trap for the mirror. The value of $\Omega$, which serves as the IR cutoff in the energy scale in this system, should be small enough comparing to $r_{b}^{z}$, which is the UV energy cutoff. This shows the qualitative difference for the theories in two regions, $1<\alpha<2$ and $2<\alpha<3$. It can be seen that when other parameters are fixed, the saturated Von-Newmann entropy reaches maximal when $\alpha$ approaches 2 . This marked the transition relates to the fact that in the IR limit the mass of the mirror is an irrelevant operator as $\alpha<2$ and a relevant operator as $\alpha>2$. However, for $\alpha>3$, it is also found that to have the Von-Newmann entropy consistent with the minimum uncertainty relation of quantum mechanical systems requires the curvature radius $L$ in the Lifshitz metric to be of the order of Planck length $l_{p}$. This implies the breakdown of the assumption in the holographic approach by treating the background geometry as the classical configuration given by the solution of Einstein equations. The quantum gravity effects need to be taken into account in this case and deserves further study.

Our presentation is organized as follows. In next section, we review the idea of open quantum systems and introduce the method of the closed-time-path formalism. The environmental degrees of freedom in the full density matrix of the system-plus-environment are traced over to obtain the reduced density matrix of the system. Environmental effects are 
then all encoded in the influence functional, which can be completely determined by the nonequilibrium two-point correlators in the linear response approximation. In section 3 , we briefly review the method of the holographic influence functional, and present the analytical form of the nonequilibrium two-point correlators to be used in the later calculation. The more detailed derivation of these correlators is in appendix. In section 4, we study the dynamics of the mirror with a harmonic trap potential, immersed in the environment of strongly coupled quantum critical fields. Before turning on the interaction of mirror with the environment, the initial state is assumed to be the direct product of the mirror's ground state and the vacuum state of the quantum critical field. When turning on the interaction, we obtain the effective nonequilibrium action by tracing out the environment fields and introducing the noise's degree of freedom. The Heisenberg equations of motion for the mirror's degree of freedom can be derived from the effective action. Their real-time solutions can be studied using the Laplace transform technique. In section 5, we obtain the time evolution of the entanglement entropy from the expectation value of $\hat{q}^{2}, \hat{p}^{2}$, and $\hat{p} \hat{q}+\hat{q} \hat{p}$, and show our main results. In section 6 , the comparison to the case with the environment fields given by the relativistic free fields is made in the paradigm of quantum Brownian motion. Concluding remarks are in section 7.

\section{Nonequilibrium effective action and influence functional}

In quantum systems, the complete information of the expectation values and correlation functions can be determined by the time dependent density matrix $\rho(t)$. The closedtime-path formalism enables us to calculate the evolution of the density matrix that has been prepared at some particular initial time $t_{i}$. In this work, we consider the system linearly coupled to an environment field. The full Lagrangian consisting of the systemplus-environment, takes the form

$$
L(q, F)=L_{q}[q]+L_{F}[F]+q F
$$

where $q$ and $F$ generically represent the system and the environment variables respectively. We assume that the initial density matrix at time $t_{i}$ can be factorized as

$$
\rho\left(t_{i}\right)=\rho_{q}\left(t_{i}\right) \otimes \rho_{F}\left(t_{i}\right)
$$

where $\rho_{F}\left(t_{i}\right)$ is the initial density matrix of the environment. The full density matrix $\rho(t)$ evolves unitarily according to

$$
\rho\left(t_{f}\right)=U\left(t_{f}, t_{i}\right) \rho\left(t_{i}\right) U^{-1}\left(t_{f}, t_{i}\right)
$$

with $U\left(t_{f}, t_{i}\right)$ the time evolution operator, involving the degrees of freedom of the system and environment. The reduced density matrix $\rho_{r}$ of the system of interest can be obtained by tracing over the environmental degrees of freedom, $F$ in the full density matrix and can be written as $[7-12]$

$$
\rho_{r}\left(q_{f}, \tilde{q}_{f}, t_{f}\right)=\int d q_{1} d q_{2} \mathcal{J}\left(q_{f}, \tilde{q}_{f}, t_{f} ; q_{1}, q_{2}, t_{i}\right) \rho_{q}\left(q_{1}, q_{2}, t_{i}\right)
$$


where the propagating function $\mathcal{J}\left(q_{f}, \tilde{q}_{f}, t_{f} ; q_{1}, q_{2}, t_{i}\right)$ is

$$
\mathcal{J}\left(q_{f}, \tilde{q}_{f}, t_{f} ; q_{1}, q_{2}, t_{i}\right)=\int_{q_{1}}^{q_{f}} \mathcal{D} q^{+} \int_{q_{2}}^{\tilde{q}_{f}} \mathcal{D} q^{-} \exp \left[i \int_{t_{i}}^{t_{f}} d t\left(L_{q}\left[q^{+}\right]-L_{q}\left[q^{-}\right]\right)\right] \mathcal{F}\left[q^{+}, q^{-}\right]
$$

with a path integral that propagates forward and backward in time. For the environment of a free field theory or in the linear response approximation, the influence functional $\mathcal{F}\left[q^{+}, q^{-}\right]$ can be written in terms of real-time Green's functions of the environment fields $F$ [6],

$$
\begin{aligned}
\mathcal{F}\left[q^{+}, q^{-}\right]= & \exp \left\{-\frac{i}{2} \int_{t_{i}}^{t_{f}} d t \int_{t_{i}}^{t_{f}} d t^{\prime}\left[q^{+}(t) G^{++}\left(t, t^{\prime}\right) q^{+}\left(t^{\prime}\right)-q^{+}(t) G^{+-}\left(t, t^{\prime}\right) q^{-}\left(t^{\prime}\right)\right.\right. \\
& \left.\left.-q^{-}(t) G^{-+}\left(t, t^{\prime}\right) q^{+}\left(t^{\prime}\right)+q^{-}(t) G^{--}\left(t, t^{\prime}\right) q^{-}\left(t^{\prime}\right)\right]\right\} .
\end{aligned}
$$

The Green's functions involved are time-ordered, anti-time-ordered and Wightman functions, defined as

$$
\begin{aligned}
& i G^{+-}\left(t, t^{\prime}\right)=\left\langle F\left(t^{\prime}\right) F(t)\right\rangle, \\
& i G^{-+}\left(t, t^{\prime}\right)=\left\langle F(t) F\left(t^{\prime}\right)\right\rangle, \\
& i G^{++}\left(t, t^{\prime}\right)=\left\langle F(t) F\left(t^{\prime}\right)\right\rangle \theta\left(t-t^{\prime}\right)+\left\langle F\left(t^{\prime}\right) F(t)\right\rangle \theta\left(t^{\prime}-t\right), \\
& i G^{--}\left(t, t^{\prime}\right)=\left\langle F\left(t^{\prime}\right) F(t)\right\rangle \theta\left(t-t^{\prime}\right)+\left\langle F(t) F\left(t^{\prime}\right)\right\rangle \theta\left(t^{\prime}-t\right),
\end{aligned}
$$

where the expectation values are calculated in the state described by $\rho_{F}\left(t_{i}\right)$. The retarded Green's function and Hadamard function can be constructed from them according to

$$
\begin{aligned}
& G_{R}\left(t-t^{\prime}\right) \equiv-i \theta\left(t-t^{\prime}\right)\left\langle\left[F(t), F\left(t^{\prime}\right)\right]\right\rangle=\left\{G^{++}\left(t, t^{\prime}\right)-G^{+-}\left(t, t^{\prime}\right)\right\}, \\
& G_{H}\left(t-t^{\prime}\right) \equiv \frac{1}{2}\left\langle\left\{F(t), F\left(t^{\prime}\right)\right\}\right\rangle=\frac{i}{4}\left\{G^{++}\left(t, t^{\prime}\right)+G^{+-}\left(t, t^{\prime}\right)+G^{--}\left(t, t^{\prime}\right)+G^{-+}\left(t, t^{\prime}\right)\right\} .
\end{aligned}
$$

In a time-translation invariant environment, the Fourier transform of various Green's functions is defined by

$$
G\left(t-t^{\prime}\right)=\int \frac{d \omega}{2 \pi} G(\omega) e^{-i \omega\left(t-t^{\prime}\right)} .
$$

Notice that the above Green's functions are not totally independent as a result of the unitarity property of the system-plus-environment. In particular, as the environment is in thermal equilibrium at the temperature $T$ initially with $\rho_{F}\left(t_{i}\right)=e^{-\frac{1}{T} H_{F}}$, the fluctuationdissipation relation gives

$$
G_{H}(\omega)=-\left(1+2 n_{\omega}\right) \operatorname{Im} G_{R}(\omega)
$$

with $n_{\omega}=\left(e^{\frac{\omega}{T}}-1\right)^{-1}$. In the next section, we briefly review the construction of Lifshitz geometry and the method of holographic influence functional, with which we explore the effects of the strongly coupled quantum critical fields on the system. 


\section{The holography model and holographic influence functional}

The environment that we will consider is described by the theory of $d$-dimensional quantum critical points with the following scaling symmetry:

$$
t \rightarrow \mu^{z} t, \quad x \rightarrow \mu x
$$

where $z$ is called the dynamical exponent. It's gravity dual is described by the $d+1$ dimensional Lifshitz geometry with the metric [39],

$$
d s^{2}=-\frac{r^{2 z}}{L^{2 z}} d t^{2}+\frac{1}{r^{2}} d r^{2}+\frac{r^{2}}{L^{2}} d x_{i} d x_{i}
$$

where the scaling symmetry (3.1) is realized as an isometry of this metric. In the following calculations we will set the curvature radius $L=1$, and restore $L$ when giving the order of magnitude estimate on the quantities of the system. This $d+1$-dimensional Lifshitz metric can be constructed by coupling gravity with negative cosmological constant to massive Abelian vector fields [45]. The influence functional for the mirror $(n \geq 1)$ can be obtained in the gravity theory by computing the on-shell DBI action of the brane. The details can be found in $[14,46]$ and also in the appendix. There we first introduce $Q^{+}\left(t, r_{1}\right)$ and $Q^{-}\left(t, r_{2}\right)$, which describe the brane's positions around the stationary configuration in two regions with different asymptotic boundaries in the maximally extended Lifshitz black hole geometry $[46,47]$. We then impose the boundary conditions at $r_{b}$

$$
q^{ \pm}(t)=Q^{ \pm}\left(t, r_{b}\right)
$$

and the analyticity conditions in the black hole horizons. By identifying the variables $q^{ \pm}(t)$ as the displacement of the moving mirror in the close-time-path formalism, the classical onshell action of the brane can then be identified as the influence functional for the mirror [46]:

$$
\mathcal{F}\left[q^{+}, q^{-}\right]=S_{\text {gravity }}\left(Q^{+}(t, r), Q^{-}(t, r)\right)=S_{\mathrm{DBI}}^{\text {on-shell }}\left(Q^{+}\right)-S_{\mathrm{DBI}}^{\text {on-shell }}\left(Q^{-}\right)
$$

where $S_{\mathrm{DBI}}^{\text {on-shell }}$ is the on-shell DBI action for the probe brane. We call this construction of $\mathcal{F}\left[q^{+}, q^{-}\right]$the holographic influence functional. Various Green's functions of the field can be read off (See appendix).

The DBI action in the case of the $n+1$-dimensional probe brane in the Lifshitz black hole, up to the quadratic order in perturbations, is written in appendix. Here we just present it in the zero temperature limit $\left(r_{h} \rightarrow 0\right)$ for the sake of introducing the notations and the assumptions, given by

$$
S_{\mathrm{DBI}} \simeq \text { constant }-\frac{T_{n+1}}{2} \int d r d t d x_{1} d x_{2} \ldots d x_{n}\left(r^{z+n+3} X^{\prime I} X^{\prime I}-\frac{\dot{X}^{I} \dot{X}^{I}}{r^{z-n-1}}\right),
$$

where $T_{n+1}$ is the tension of the brane and $X^{I}(t, r)$ parameterizes the brane's position around the stationary configuration, where $I=n+1, \ldots, d$ denotes the transverse directions to the brane. Also, $X^{I}=\partial_{r} X^{I}, \dot{X}^{I}=\partial_{t} X^{I}$. We assume that the mirror does not deform when moving in its transverse directions so that all $X^{I}$ depends only on $t$ and $r$. In 
the quadratic order, the perturbations in different directions decouple, and a particular direction $X^{I}$ is considered. Again, we start from the DBI action for the probe brane in the Lifshitz black hole, from which to be able to determine $Q^{ \pm}$in two outside regions of the black hole separately. In the zero temperature limit by taking $r_{h} \rightarrow 0$, the zero-temperature retarded Green's function with $\alpha=1+(n+2) / z$, for $\omega>0$ can be found to be,

$$
G_{R}(\omega)=-T_{n+1} S_{n} \omega r_{b}^{n+2} \frac{H_{\frac{\alpha}{2}-1}^{(1)}\left(\frac{\omega}{z r_{b}^{z}}\right)}{H_{\frac{\alpha}{2}}^{(1)}\left(\frac{\omega}{z r_{b}^{z}}\right)},
$$

where $H_{\nu}^{(1)}(x)$ is the Hankel function of the first kind and $S_{n}$ is the volume of the mirror. As we will particularly focus on the late time dynamics, the small $\omega$ expansion of $G_{R}(\omega)$ is considered,

$$
G_{R}(\omega)=m(i \omega)^{2}+\gamma(-i \omega)^{\alpha}+\mathcal{O}\left(\omega^{2} / r_{b}^{2 z}\right)
$$

where

$$
m=\frac{T_{n+1} S_{n}}{z(\alpha-2) r_{b}^{z(2-\alpha)}},
$$

and

$$
\gamma=\frac{T_{n+1} S_{n}}{(2 z)^{\alpha-1}} \frac{\Gamma\left(1-\frac{\alpha}{2}\right)}{\Gamma\left(\frac{\alpha}{2}\right)} .
$$

In this expansion there are the terms of even powers in $\omega$ to be treated as the correction to the dispersion relation. We regard the $\omega^{\alpha}$ term as the leading contribution to the damping effect. Apparently, the interaction between the mirror and quantum critical fields not only gives the mirror's mass $m$ with the $r_{b}$ dependence but also induce the damping effect on the mirror with the coefficient $\gamma$. The friction coefficient $\gamma$ is independent of $r_{b}$, which becomes important in stabilizing the dynamics of the mirror in a fluctuating environment. The zero-temperature Hadamard function for $\omega>0$ is found to be,

$$
G_{H}(\omega)=\frac{2 z}{\pi} r_{b}^{n+2+z} \frac{T_{n+1} S_{n}}{J_{\frac{\alpha}{2}}^{2}\left(\frac{\omega}{z r_{b}^{z}}\right)+Y_{\frac{\alpha}{2}}^{2}\left(\frac{\omega}{z r_{b}^{z}}\right)},
$$

where $J_{\nu}(x)$ and $Y_{\nu}(x)$ are Bessel functions. Also, in the small $\omega$ limit, the Hadamard function can be approximated by

$$
G_{H}(\omega)=\frac{\pi T_{n+1} S_{n}}{(2 z)^{\alpha-1} \Gamma^{2}\left(\frac{\alpha}{2}\right)} \omega^{\alpha}+\mathcal{O}\left(\omega^{\alpha+2} / r_{b}^{z(\alpha+2)}\right) .
$$

Notice that the obtained retarded Green's function and the Hadamard function obey the zero-temperature fluctuation-dissipation theorem.

\section{Dynamics of a moving mirror}

Let us now specify the Lagrangian of the system. We see from the previous section that the interaction with the environment field gives the mass correction $m$ (3.8) to the mirror in the small $\omega$ limit. We here introduce the bare mass $m_{0}$ where the renormalized mass 
becomes $M=m+m_{0}$. To give the system an infrared energy scale, we also add a quadratic trap potential with the oscillation frequency $\Omega_{0}$ to the mirror. Thus, the Lagrangian for the mirror with the displacement $q$ can be written as

$$
L_{q}[q]=\frac{1}{2} m_{0} \dot{q}^{2}-\frac{1}{2} m_{0} \Omega_{0}^{2} q^{2} .
$$

The frequency $\Omega_{0}$ is set to be small comparing to the UV cutoff $r_{b}^{z}$, and plays a role as an IR cutoff. The retarded Green's function in (3.6) after subtracting the mass correction term $m$ is defined to be the self-energy $\Sigma(\omega)$

$$
\Sigma(\omega)=G_{R}(\omega)-m(i \omega)^{2} .
$$

To study the stochastic dynamics of the system, it is more convenient to change the $q^{+}, q^{-}$coordinates to the average and relative coordinates:

$$
q=\left(q^{+}+q^{-}\right) / 2, \quad q_{\Delta}=q^{+}-q^{-} .
$$

Thus, the coarse-grained effective action can be defined from (2.5) by using the Lagrangian of the mirror in (4.1) and the holographic influence functional in (2.6), as

$$
\begin{aligned}
S_{\mathrm{CG}} & {\left[q^{ \pm}=q \pm \frac{q_{\Delta}}{2}\right]=\int_{t_{i}}^{t_{f}} d t\left(L_{q}\left[q^{+}\right]-L_{q}\left[q^{-}\right]\right)-i \ln \mathcal{F}\left[q^{+}, q^{-}\right] } \\
= & \int_{t_{i}}^{t_{f}} d t \int_{t_{i}}^{t_{f}} d t^{\prime}\left(M \dot{q}_{\Delta}(t) \dot{q}\left(t^{\prime}\right)-M \Omega^{2} q_{\Delta}(t) q\left(t^{\prime}\right)\right)-\int_{t_{i}}^{t_{f}} d t \int_{t_{i}}^{t} d t^{\prime} q_{\Delta}(t) \Sigma\left(t-t^{\prime}\right) q\left(t^{\prime}\right) \\
& +\frac{i}{2} \int_{t_{i}}^{t_{f}} d t \int_{t_{i}}^{t_{f}} d t^{\prime} q_{\Delta}(t) G_{H}\left(t-t^{\prime}\right) q_{\Delta}\left(t^{\prime}\right),
\end{aligned}
$$

where $G_{H}(t)$ and $\Sigma(t)$ are the inverse Fourier transform of $G_{H}(\omega)$ and $\Sigma(\omega)$, and $\Omega=\left(1-\frac{m}{M}\right) \Omega_{0}$. Apparently, the relevant Green's functions that contribute to the mirror's dynamics at quadratic order are the retarded Green's function and Hadamard function, which we have obtained above in the holographic setup. After tracing over the degrees of freedom of the environment, the system of the interest becomes non-unitary, and the resulting coarse-grained effective action becomes complex-valued function. Before turning on the interaction with the environment, the initial density matrix of the mirror is assumed to be in its ground state of a simple harmonic oscillator with frequency $\Omega$,

$$
\rho_{q}\left(q, q^{\prime} ; t_{i}\right)=\left(\frac{M \Omega}{\pi}\right)^{1 / 2} e^{-\left[M \Omega^{2}\left(q^{2}+q^{\prime 2}\right)\right]}
$$

where the initial position and momentum uncertainties are given respectively by $(\Delta q)^{2}\left(t_{i}\right)=(2 M \Omega)^{-1}$ and $(\Delta p)^{2}\left(t_{i}\right)=\frac{1}{2} M \Omega$. It is possible to carry out the path integral over the mirror's degree of freedom in (2.5) and derive the reduced density of the matrix at time $t$. Then, the entanglement entropy of the system can be computed by taking the trace of the reduced density matrix with respect to the degrees of freedom of the mirror via $S=-\operatorname{Tr}_{q}\left[\rho_{r} \ln \rho_{r}\right]$. Instead of resorting to this straightforward but cumbersome method, we use the fact that, in the linear response approximation, the resulting reduced density 
matrix, with the initial condition in (4.5), remains Gaussian when the system is linearly coupled to the environment. ${ }^{1}$ Then the entanglement entropy can also be computed from the expectation values of $\hat{q}^{2}, \hat{p}^{2}$, and $\hat{q} \hat{p}+\hat{p} \hat{q}$, where $\hat{q}$ and $\hat{p}$ are respectively the position and the momentum operators of the mirror [48]. To find the time dependent expectation values, we need to derive the equation of motion for the position operator, incorporating the effects from the environment. This can be done by introducing an auxiliary variable $\eta(t)$, the noise force, with a Gaussian distribution function:

$$
P[\eta(t)]=\exp \left\{-\frac{1}{2} \int_{t_{i}}^{t_{f}} d t \int_{t_{i}}^{t_{f}} d t^{\prime} \eta(t) G_{H}^{-1}\left(t-t^{\prime}\right) \eta\left(t^{\prime}\right)\right\} .
$$

In terms of the noise force $\eta(t), S_{C G}$ can be rewritten as an ensemble average over $\eta(t)$,

$$
\exp i S_{C G}=\int \mathcal{D} \eta P[\eta(t)] \exp i S_{\eta}\left[q, q_{\Delta} ; \eta\right],
$$

where the stochastic coarse-grained effective action $S_{\eta}$ is given by

$$
\begin{aligned}
S_{\eta}\left[q, q_{\Delta} ; \eta\right]= & \int_{t_{i}}^{t_{f}} d t \int_{t_{i}}^{t_{f}} d t^{\prime}\left(M \dot{q}_{\Delta}(t) \dot{q}\left(t^{\prime}\right)-M \Omega^{2} q_{\Delta}(t) q\left(t^{\prime}\right)\right) \\
& -\int_{t_{i}}^{t_{f}} d t \int_{t_{i}}^{t} d t^{\prime} q_{\Delta}(t) \Sigma\left(t-t^{\prime}\right) q\left(t^{\prime}\right)+\int_{t_{i}}^{t_{f}} \eta(t) q_{\Delta}(t) .
\end{aligned}
$$

Then, in the canonical formalism, the equation of motion of the position operator is found to be,

$$
M \ddot{\hat{q}}(t)+M \Omega^{2} \hat{q}(t)+\int_{0}^{t} \Sigma\left(t-t^{\prime}\right) \hat{q}\left(t^{\prime}\right) d t^{\prime}=\hat{\eta}(t)
$$

with the initial time set to be $t_{i}=0$. There are two different sources of quantum fluctuations, over which the averages are taken on mirror's position. One is the average over intrinsic quantum fluctuations of the mirror, and the other is the average over the noise manifested from quantum fluctuations of the environment. The noise distribution function $P[\eta(t)]$ in (4.6) leads to the correlation function of the noise as follows

$$
\left\langle\hat{\eta}(t) \hat{\eta}\left(t^{\prime}\right)\right\rangle=G_{H}\left(t-t^{\prime}\right) .
$$

For the environment consisting of a collection of harmonic oscillators, the Heisenberg equations of motion of the system and the environment can be solved exactly, giving the same form of the above equation with the associated Green's functions constructed out of the harmonic oscillators $[4,5,49]$. In this work, the influence functional is derived holographically, and in this way the retarded Green's function and Hadamard function contains the information of the strongly coupled quantum critical fields.

The solutions of (4.9) can be expressed in term of the fundamental solutions $G_{1}(t)$ and $G_{2}(t)$, defined to be the solutions of the homogeneous equations without the noise term, obeying the following boundary conditions $[12,49]$ :

$$
\begin{aligned}
& G_{1}(0)=1, \quad \dot{G}_{1}(0)=0 ; \\
& G_{2}(0)=0, \quad \dot{G}_{2}(0)=1 .
\end{aligned}
$$

\footnotetext{
${ }^{1}$ We thank Jen-Tsung Hsiang for pointing out this trick to us.
} 
The Laplace transform of them is respectively defined as

$$
G_{1,2}(s)=\int_{0}^{\infty} G_{1,2}(t) e^{-s t} d t
$$

Then $G_{1,2}(s)$ can be found to be

$$
\begin{aligned}
& G_{1}(s)=\frac{s}{s^{2}+\Omega^{2}+\tilde{\Sigma}(s)} \\
& G_{2}(s)=\frac{1}{s^{2}+\Omega^{2}+\tilde{\Sigma}(s)}
\end{aligned}
$$

with the function $\tilde{\Sigma}(s) \equiv \Sigma(-i s) / M$, where $\Sigma(\omega)$ is the self-energy of the mirror (4.2). The solution of $\hat{q}(t)$ can be expressed in terms of $G_{1}(t)$ and $G_{2}(t)$ as

$$
\hat{q}(t)=G_{1}(t) \hat{q}(0)+G_{2}(t) \frac{\hat{p}(0)}{M}+\frac{1}{M} \int_{0}^{t} d t^{\prime} G_{2}\left(t-t^{\prime}\right) \hat{\eta}\left(t^{\prime}\right) .
$$

The real-time function of $G_{1,2}$ is given by the inverse Laplace transform

$$
G_{1,2}(t)=\frac{1}{2 \pi i} \int_{C} e^{s t} G_{1,2}(s) d s
$$

where $C$ refers to the Browmwich contour running along the imaginary axis to the right of all the poles and cuts of $G_{1,2}(s)$ in the complex s plane. Therefore we need to understand the analytical structure of the self-energy $\Sigma$ to obtain the real-time dynamics of all expectation value of the mirror's variables. Here we will mainly focus on the late time dynamics of the mirror to find its relaxation dynamics as well as saturation behavior on the lose of information about the initial conditions of the system influenced by the environment. Accordingly, the retarded Green's function and Hadamard function in their small- $\omega$ approximation in (3.7) and (3.11) will be applied. In principle, we can study the early time behavior since the exact form of the Green's functions is known. However, due to the complicated analytical structure of the self energy, we will leave it for the future study.

From the expression (3.7) for the small- $\omega$ approximation of the retarded Green's function, the function $\tilde{\Sigma}(s)$ is approximated by

$$
\tilde{\Sigma}(s) \simeq \frac{\gamma}{M} s^{\alpha} .
$$

For non-integer values of $\alpha$, we choose a branch cut on the negative real $s$ axis. This amounts to choosing that

$$
\tilde{\Sigma}\left(s=-s_{0} \pm i \epsilon\right)=\operatorname{Re} \tilde{\Sigma}\left(-s_{0}\right) \pm i \operatorname{Im} \tilde{\Sigma}\left(-s_{0}\right),
$$

for $s_{0}>0$, where we define

$$
\begin{aligned}
& \operatorname{Re} \tilde{\Sigma}\left(-s_{0}\right)=\frac{\gamma}{M} s_{0}^{\alpha} \cos (\alpha \pi), \\
& \operatorname{Im} \tilde{\Sigma}\left(-s_{0}\right)=\frac{\gamma}{M} s_{0}^{\alpha} \sin (\alpha \pi) .
\end{aligned}
$$


Notice that the same form of the self-energy has also been studied in the works $[50,51]$ where the system is coupled to the bath of harmonic oscillators. The properties of the bath is completely characterized by the spectral density that has the form, for example, $J(\omega) \propto \omega^{\beta}$ for $\omega>0$ with non-integer $\beta$, where $\omega$ is the frequency of oscillators. Also notice that the branch cuts arise due to the fact that the parameter $\alpha=1+(n+2) / z$ is a non-integer. Apart from the cuts, there exist the poles determined by

$$
s_{p}^{2}+\Omega^{2}+\tilde{\Sigma}\left(s_{p}\right)=0
$$

where the real and imaginary parts of $s_{p}$ correspond to the oscillatory frequency and the decay rate, and we denote them by

$$
s_{p}= \pm i \omega_{p}-\Gamma
$$

Thus these states of the mirror are not exactly the eigenstates of the interacting Hamiltonian. To have the analytical expressions, we consider two separate limiting situations with regard to the relative importance of the kinetic term $s^{2}$ to the damping term $\Sigma(s) \simeq \frac{\gamma}{M} s^{\alpha}$ at the positions of the poles. These two limiting cases are characterized by the parameter, $\Delta \equiv \frac{\gamma}{M} \Omega^{\alpha-2}$, where $|\Delta| \ll 1$ is for the kinetic term dominance and $|\Delta| \gg 1$ is for the damping term dominance. Using (3.9), the parameter $\Delta$ can also be written as,

$$
\Delta=\frac{T_{n+1} \pi \Omega^{\alpha-2}}{(2 z)^{\alpha-1} \rho_{n} \Gamma^{2}\left(\frac{\alpha}{2}\right) \sin \frac{\pi \alpha}{2}}
$$

with the mass density of the mirror, $\rho_{n}=M / S_{n}$. As we will see later, the runaway solutions with negative $\Gamma$ exist for $\alpha>2$. From the field theory perspective, the problem of the existence of the runaway solutions is due to the fact that the resulting equation involves higher than 2nd-order time derivative terms, giving the unpleasant feature of instability. For example, the Abraham-Lorentz equation, which includes the radiation reaction force for a nonrelativistic charged particle, is third-order in time and possesses runaway solutions. In electrodynamics, the problem can be solved [52, chapter 17.14] by reformulating the theory to eliminate unstable solutions with the method of reduction of order that reduces the equation to the 2 nd-order. The similar procedure also works in the Einstein gravity $[53,54]$. Here we assume all the runway solutions can be removed by the above mentioned method and discuss stable solutions only. In case that there are more than one stable mode, we concentrate on the most long-lived mode to be discussed in the following section. Furthermore, as $\alpha>3$, we will see the breakdown of our assumption of the Gaussian reduced density matrix from the obtained saturated entanglement entropy, which shows the mass density of the mirror needs to be of the Planck scale order, being consistent with the minimum uncertainty of quantum systems. Thus in the following discussion, we will focus on the damping term dominated region, $1<\alpha<2$ and the kinetic term dominated region, $2<\alpha<3$ respectively. In these cases, the Browmwich contour for the inverse Laplace transform in (4.16) can be deformed as in figure 1. We will discuss the contributions from the poles and the branch cut separately. 


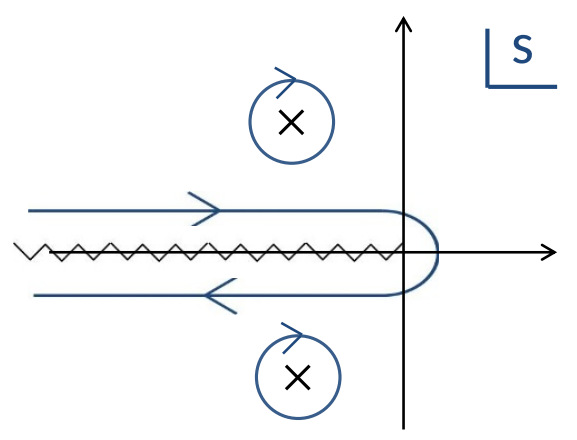

Figure 1. The contour for the inverse Laplace transform used to compute the fundamental solutions $G_{1}(t)$ and $G_{2}(t)$ with the existence of the cut and the poles.

\subsection{The pole contributions}

We now restore the curvature radius $L$ for the order-of-magnitude estimation of relevant quantities. Then the parameter $\Delta \simeq\left(T_{n+1} L^{n+2}\right)\left(L^{n+1} \rho_{n}\right)^{-1}(L \Omega)^{\alpha-2}$. In the top-down constructions, the tension of the branes, which is related to the string coupling, determines the Planck length scale $l_{p}$ in the gravity theory by $\left(T_{n+1} L^{n+2}\right) \simeq\left(\frac{L}{l_{p}}\right)^{n+2}$. In the classical gravity limit (corresponding to the large- $N$ limit in the boundary theory), we should have $\left(T_{n+1} L^{n+2}\right) \gg 1$. Also, in the boundary theory, we may choose the unit such that $\rho_{n} \simeq L^{-n-1}$. If so, the condition $\Delta \gg 1$ for the damping term dominated can be achieved for all values of $\alpha$ by choosing $\Omega \simeq L^{-1}$. As for $\Delta \ll 1$ in the case of the kinetic term dominated, the choice of $\Omega$ has to satisfy $(L \Omega)^{\alpha-2} \ll\left(\frac{l_{p}}{L}\right)^{n+2}$. In the following, we will discuss the late time behavior of the mirror in these two limits separately.

\subsubsection{The damping term dominated region $(\Delta \gg 1)$}

As $\Delta \gg 1$, the resonance frequency $\omega_{p}$ and the width $\Gamma$ can be found for $1<\alpha<2$ to be

$$
\begin{aligned}
\omega_{p} & \simeq \Omega \Delta^{-\frac{1}{\alpha}} \sin \frac{\pi}{\alpha}, \\
\Gamma & \simeq-\Omega \Delta^{-\frac{1}{\alpha}} \cos \frac{\pi}{\alpha} .
\end{aligned}
$$

The width $\Gamma$ is positive for $1<\alpha<2$, corresponding to the stable solution. Moreover they are the only poles in the principal sheet. As for $2<\alpha<3$, there are more than two poles on the principal sheet and also the runaway solutions with $\Gamma<1$ may exist. We will assume that these unstable modes can be removed by the reduction of order method as described in the previous section, and concentrate on the stable mode. In this case, the stable mode is found to be

$$
\begin{aligned}
\omega_{p} & \simeq \Omega(-\Delta)^{-\frac{1}{\alpha}} \sin \frac{2 \pi}{\alpha}, \\
\Gamma & \simeq-\Omega(-\Delta)^{-\frac{1}{\alpha}} \cos \frac{2 \pi}{\alpha} .
\end{aligned}
$$

These poles contribute to the functions $G_{1,2}(t)$ by

$$
\begin{aligned}
& G_{2, \text { pole }}(t) \propto \cos \left[\omega_{p} t+\varphi\right] e^{-\Gamma t}, \\
& G_{1, \text { pole }}(t)=\frac{d}{d t} G_{2, \text { pole }}(t),
\end{aligned}
$$


where $\varphi$ is a constant. This solution is valid when $t \gg r_{b}^{-z}$. Since the resonance frequency and the width are of the same order, it leads to the broad resonance behavior as in contrast with the narrow resonance cases that will occur in the kinetic term dominated region.

\subsubsection{The kinetic term dominated region $(\Delta \ll 1)$}

As $\Delta \ll 1$, we find the narrow resonance modes of the long-lived resonances, which correspond to an almost energy eigenstate of the interactive Hamiltonian with the width much smaller than the oscillation frequency. The frequency and the width are found perturbatively to be

$$
\omega_{p}=\Omega+\delta \Omega
$$

where

$$
\delta \Omega=\frac{1}{2} \Omega \Delta \cos \left(\frac{\alpha \pi}{2}\right)
$$

and

$$
\Gamma=\frac{1}{2} \Omega \Delta \sin \left(\frac{\alpha \pi}{2}\right)
$$

with $\Gamma>0$ in both the ranges, $1<\alpha<2$ and $2<\alpha<3$. However as $2<\alpha<3$, there are runaway solutions in the principal sheet, which are presumably removed. No matter it is narrow or broad resonance, the pole contributions to the functions $G_{1}(t)$ and $G_{2}(t)$ decay exponentially in general as in (4.26). In the following, we consider the cut contributions and discuss the time scales, after which $G_{1}(t)$ and $G_{2}(t)$ will relax in power-law behavior in stated.

\subsection{The cut contributions}

The cut contributions to the $G_{1,2}$ functions can be expressed as

$$
\begin{aligned}
& G_{2, \text { cut }}(t)=-\frac{1}{\pi} \int_{0}^{\infty} d s \frac{\operatorname{Im} \tilde{\Sigma}(-s)}{\left[s^{2}+\Omega^{2}+\operatorname{Re} \tilde{\Sigma}(-s)\right]^{2}+[\operatorname{Im} \tilde{\Sigma}(-s)]^{2}} e^{-s t}, \\
& G_{1, \text { cut }}(t)=\frac{d}{d t} G_{2, \text { cut }}(t),
\end{aligned}
$$

where $\operatorname{Re} \tilde{\Sigma}$, and $\operatorname{Im} \tilde{\Sigma}$ are defined in (4.19) and (4.20) respectively. Notice that in obtaining this expression, we have already used the small $\omega$ approximation $\left(\frac{\omega}{r_{b}^{z}} \ll 1\right)$ for $\Sigma(\omega)$, to focus on the late time behavior, namely $t \gg r_{b}^{-z}$.

Since the integral for the cut contributions is dominated by the small $s$ region in the late times, simpler results can be obtained for much latter times, $t \gg \Omega^{-1}$, comparing to the late time region $\left(t \gg r_{b}^{-z}\right)$, which are the time scales for the pole contributions dominated. In this case, the integrand behaves like $s^{\alpha} e^{-s t}$ and the integral can be evaluated by the Gamma function to be

$$
G_{2, \mathrm{cut}}(t) \simeq \frac{\Delta}{\pi \Omega} \Gamma(1+\alpha) \sin (\alpha \pi)(\Omega t)^{-1-\alpha} .
$$

The function $G_{1, \text { cut }}(t)$ is just the time derivative of $G_{2 \text {,cut }}(t)$. Naively, it may look like that the cut contributions dominate over the pole contributions as $t>\Gamma^{-1}$, which is the 


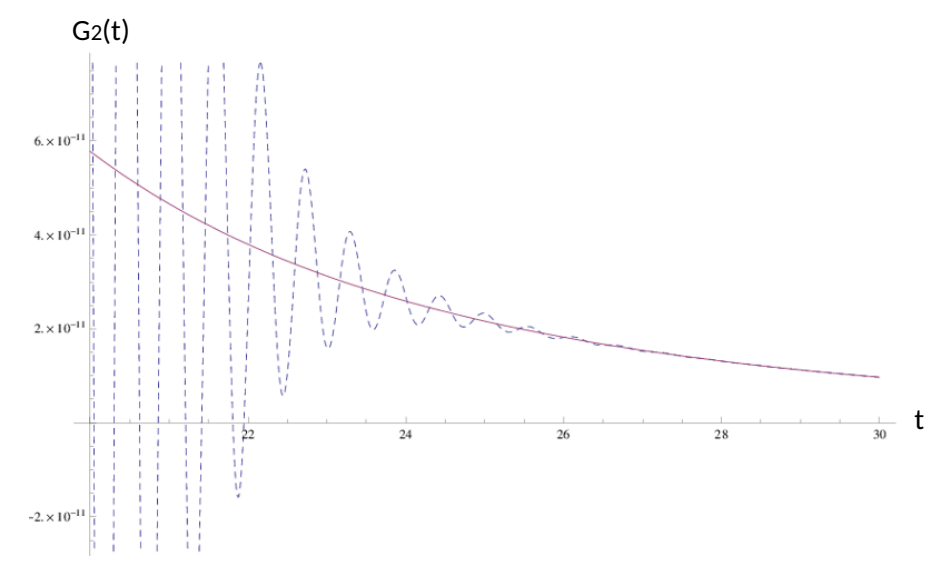

Figure 2. The dash line shows the numerical result for $G_{2}(t)$ from the poles and cut contributions with their expressions (See main text for details) by setting $\alpha=2.4, \Omega=10, \frac{\gamma}{M}=-0.01$, and $T_{n+1} S_{n}=15$. We also set $\Delta=\frac{\gamma}{M} \Omega^{\alpha-2}=-0.25$, which corresponds to the case of narrow resonance. The solid line is also for the evolution of $G_{2}(t)$ in the late times $(t \gg 1 / \Omega)$ given by (4.33) with the same parameters above.

decay time. However, after comparing $G_{2 \text {,cut }}(t)$ with $G_{2 \text {,pole }}(t)$, we find that in fact for the power-law behavior of cut contributions to dominate, the relevant time scales are $t \gtrsim t_{n} \equiv$ $-\Omega^{-1}(\alpha+1) \Delta^{-1} \ln \Delta$ for $\Delta \ll 1$ (narrow resonance) and $t \gtrsim t_{b} \equiv \Omega^{-1}(1+1 / \alpha) \Delta^{1 / \alpha} \ln \Delta$ for $\Delta \gg 1$ (broad resonance).

In the following, we numerically check that the function $G_{2}(t)$ approaches $G_{2, \text { cut }}(t)$ found in (4.33). The numerics is done by doing $s$-integral in (4.31) with $\operatorname{Re} \tilde{\Sigma}(-s)$ and $\operatorname{Im} \tilde{\Sigma}(-s)$ as given in (4.19) and (4.20), and add the pole contributions given by (4.26). The results are shown in figure 2 with the chosen parameters for narrow resonance. It can be seen that $G_{2}(t)$ is oscillatory in time with the frequency $\omega_{p}$ and its amplitude decays exponentially at a rate determined by $\Gamma$. After the time $t \simeq t_{n}$, the $G_{2}(t)$ function becomes the power-law decay given by (4.33). It is interesting to contrast with the broad resonance as shown in figure 3 by choosing appropriate parameters. The $G_{2}(t)$ function in this case, is also oscillatory with smaller frequency, and after the time $t \simeq t_{b}$, it exhibits the power-law relaxation as given by the cut contributions. The $G_{1}(t)$ function can be similarly checked.

\section{Quantum uncertainties on the mirror and the entanglement entropy}

Before turning on the interaction with the environment, the initial state of the system is prepared to be in the ground state of the harmonic oscillator. The environment fields are also in zero-temperature ground state. The full density matrix is the direct product of the states of the system and the environment field, which is a pure state. The interaction with the environment turns the reduced density matrix for the system into a mixed state. However in the linear response approximation and with the bilinear coupling between the system and the environment, the reduced density matrix for the system remains Gaussian at all times. If so, the Von-Neumann entanglement entropy constructed from the Gaussian 


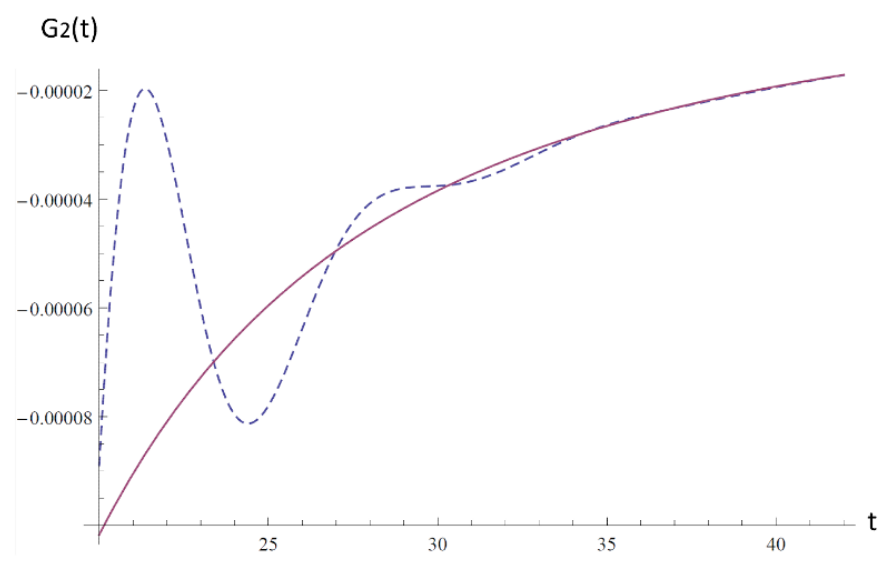

Figure 3. The dash line shows the numerical result for $G_{2}(t)$ from the poles and cut contributions with their expressions (See main text for details) by setting $\alpha=1.4, \Omega=1.5, \frac{\gamma}{M}=1.8$, and $T_{n+1} S_{n}=15$. We also set $\Delta=\frac{\gamma}{M} \Omega^{\alpha-2}=2.2$, which corresponds to the case of broad resonance. The solid line is also for the evolution of $G_{2}(t)$ in the late times $(t \gg 1 / \Omega)$ given by (4.33) with the same parameters above.

reduced density matrix can be written as [48]

$$
S(t)=\left(\sqrt{w(t)}+\frac{1}{2}\right) \ln \left(\sqrt{w(t)}+\frac{1}{2}\right)-\left(\sqrt{w(t)}-\frac{1}{2}\right) \ln \left(\sqrt{w(t)}-\frac{1}{2}\right),
$$

where the time dependent function, $w(t)$ is defined by the position and momentum uncertainties and their cross correlation,

$$
w(t) \equiv\left\langle\hat{q}^{2}(t)\right\rangle\left\langle\hat{p}^{2}(t)\right\rangle-\frac{1}{2}(\langle\hat{q}(t) \hat{p}(t)+\hat{p}(t) \hat{q}(t)\rangle)^{2} .
$$

Thus in the Gaussian state, the entanglement entropy can be obtained by calculating these three functions.

Using (4.15), the position uncertainty at time $t$ can be expressed as

$$
\begin{aligned}
\left\langle\hat{q}^{2}(t)\right\rangle & =\left\langle\hat{q}^{2}(t)\right\rangle_{I}+\left\langle\hat{q}^{2}(t)\right\rangle_{F} \\
\left\langle\hat{q}^{2}(t)\right\rangle_{I} & =G_{1}^{2}(t)\left\langle\hat{q}^{2}(0)\right\rangle+\left(G_{2}^{2}(t) / M^{2}\right)\left\langle\hat{p}^{2}(0)\right\rangle+\left(G_{1}(t) G_{2}(t) / M\right)\langle\hat{q}(0) \hat{p}(0)+\hat{p}(0) \hat{q}(0)\rangle \\
\left\langle\hat{q}^{2}(t)\right\rangle_{F} & =\frac{1}{2 M^{2}} \int_{0}^{t} d \tau \int_{0}^{t} d \tau^{\prime} G_{2}(t-\tau) G_{2}\left(t-\tau^{\prime}\right)\left\langle\left\{\hat{\eta}(\tau), \hat{\eta}\left(\tau^{\prime}\right)\right\}\right\rangle,
\end{aligned}
$$

where $\left\langle\hat{q}^{2}(t)\right\rangle_{I}$ is the uncertainty due to the intrinsic quantum fluctuations of the mirror, and thus depends on the initial position and momentum uncertainties. However $\left\langle\hat{q}^{2}(t)\right\rangle_{F}$ is the uncertainty induced by the environment and involves the two-point function of the environment field. Using the late time expressions for $G_{1}(t)$ and $G_{2}(t)\left(t \gg t_{n}\right.$ for narrow resonance and $t \gg t_{b}$ for broad resonance) obtained in last section, the various terms in $\left\langle\hat{q}^{2}(t)\right\rangle_{I}$ show the power-law relaxation:

$$
\begin{aligned}
G_{1}^{2}(t)\left\langle\hat{q}^{2}(0)\right\rangle & \propto t^{-4-2 \alpha}, \\
\left(G_{2}^{2}(t) / M^{2}\right)\left\langle\hat{p}^{2}(0)\right\rangle & \propto t^{-2-2 \alpha}, \\
\left(G_{1}(t) G_{2}(t) / M\right)\langle\hat{q}(0) \hat{p}(0)+\hat{p}(0) \hat{q}(0)\rangle & \propto t^{-3-2 \alpha} .
\end{aligned}
$$


We will see in the following that $\left\langle\hat{q}^{2}(t)\right\rangle_{I}$ depending on the initial conditions decay more quickly at late times as comparing to $\left\langle\hat{q}^{2}(t)\right\rangle_{F}$ due to the environment-induced uncertainty. The term $\left\langle\hat{q}^{2}(t)\right\rangle_{F}$ can be further expressed by $\left\langle\hat{q}^{2}(\infty)\right\rangle_{F}$, the saturated value, and $\left\langle\hat{q}^{2}(t)\right\rangle_{F, \text { asy }}$ vanishing at $t \rightarrow \infty$ as

$$
\begin{aligned}
\left\langle\hat{q}^{2}(t)\right\rangle_{F} & =\frac{1}{2 m^{2}} \int_{0}^{t} d \tau \int_{0}^{t} d \tau^{\prime} G_{2}(t-\tau) G_{2}\left(t-\tau^{\prime}\right)\left\langle\left\{\eta(\tau), \eta\left(\tau^{\prime}\right)\right\}\right\rangle \\
& =\frac{2}{m^{2}} \int_{0}^{\infty} \frac{d \omega}{2 \pi} G_{H}(\omega)\left\{\left[\int_{0}^{\infty} d \tau-\int_{t}^{\infty} d \tau\right] G_{2}(\tau) e^{-i \omega \tau}\right. \\
& {\left.\left[\int_{0}^{\infty} d \tau^{\prime}-\int_{t}^{\infty} d \tau^{\prime}\right] G_{2}\left(\tau^{\prime}\right) e^{i \omega \tau^{\prime}}\right\} } \\
& \equiv\left\langle\hat{q}^{2}(\infty)\right\rangle_{F}+\left\langle\hat{q}^{2}(t)\right\rangle_{F, \text { asy }} .
\end{aligned}
$$

The saturated value is determined by $G_{H}(\omega)$ in (3.10) and the Fourier transform of $G_{2}(t)$, which can be obtained from $G_{2}(s)$ in (4.14) by substituting $s=i \omega$ as

$$
G_{2}(\omega)=\frac{1}{-\omega^{2}+\Omega^{2}+\operatorname{Re} \Sigma(\omega) / M+i \operatorname{Im} \Sigma(\omega) / M} .
$$

The self-energy $\Sigma(\omega)$ is defined in (4.2) and for $\Omega \ll r_{b}^{z}$ we have,

$$
\operatorname{Re} \tilde{\Sigma}(\omega) \equiv \operatorname{Re} \Sigma(\omega) / M \simeq \frac{\gamma}{M} \omega^{\alpha} \cos \frac{\pi \alpha}{2}, \quad \operatorname{Im} \tilde{\Sigma}(\omega) \equiv \operatorname{Im} \Sigma(\omega) / M \simeq \frac{\gamma}{M} \omega^{\alpha} \sin \frac{\pi \alpha}{2} .
$$

Then we can write the saturation value as

$$
\begin{aligned}
\left\langle\hat{q}^{2}(\infty)\right\rangle_{F} & =\frac{2}{M^{2}} \int_{0}^{z r_{b}^{z}} \frac{d \omega}{2 \pi} G_{H}(\omega)\left|G_{2}(\omega)\right|^{2} \\
& =\frac{2}{M^{2}} \int_{0}^{z r_{b}^{z}} \frac{d \omega}{2 \pi} \frac{G_{H}(\omega)}{\left[-\omega^{2}+\Omega^{2}+\operatorname{Re} \tilde{\Sigma}(\omega)\right]^{2}+[\operatorname{Im} \tilde{\Sigma}(\omega)]^{2}} .
\end{aligned}
$$

The integral has a UV-cutoff at $\omega_{U V}=z r_{b}^{z}$. With the proper rescaling of $x \equiv \frac{\omega}{z r_{b}^{z}}$ and $\delta \equiv \frac{\Omega}{z r_{b}^{z}}$, we first consider $\delta=0$ in the limit of $\Omega \ll r_{b}^{z}$. Using the exact expression for $\Sigma(\omega)$ in $(4.2)$ and $G_{H}(\omega)$ in (3.10), it is found that

$$
\left\langle\hat{q}^{2}(\infty)\right\rangle_{F, \delta=0}=\frac{r_{b}^{z(1-\alpha)}}{\pi T_{n+1} S_{n}} \int_{0}^{1} d x \frac{g(x)}{\left|B x^{2}+x f(x)\right|^{2}}
$$

where

$$
f(x)=\frac{H_{\frac{\alpha}{2}-1}^{(1)}(x)}{H_{\frac{\alpha}{2}}^{(1)}(x)}, \quad g(x)=\frac{1}{J_{\frac{\alpha}{2}}^{2}(x)+Y_{\frac{\alpha}{2}}^{2}(x)}, \quad B=\frac{1}{\alpha-2}-\frac{z \rho_{n} r_{b}^{z(2-\alpha)}}{T_{n+1}} .
$$

The above integrant goes like $x^{\alpha-4}$ for small $x$. The integral (5.9) converges for $\alpha>3$, and thus $\left\langle\hat{q}^{2}(\infty)\right\rangle_{F, \delta=0}$ gives the leading result to the saturated value $\left\langle\hat{q}^{2}(\infty)\right\rangle_{F}$ as $\delta \rightarrow 0$. However, the integral (5.9) diverges for $1<\alpha<3$. By keeping $\delta$ finite but small, the main contributions to the integral (5.8) come from the small $x$ region, which can be approximated by

$$
\left\langle\hat{q}^{2}(\infty)\right\rangle_{F} \simeq \frac{\pi r_{b}^{z(\alpha-3)} T_{n+1}}{z^{2} S_{n} 2^{\alpha} \rho_{n}^{2} \Gamma^{2}\left(\frac{\alpha}{2}\right)} H_{\alpha}(\delta)
$$


with

$$
H_{\alpha}(\delta) \equiv \int_{0}^{1} d x \frac{x^{\alpha}}{\left(-x^{2}+\delta^{2}\right)^{2}+2 A \cos \left(\frac{\pi \alpha}{2}\right) x^{\alpha}\left(-x^{2}+\delta^{2}\right)+A^{2} x^{2 \alpha}}
$$

and $A=\frac{\pi \alpha T_{n+1} r_{b}^{z(\alpha-2)}}{z \rho_{n}}$ where the small $\omega$ expressions for $G_{H}(\omega)$ and $\Sigma(\omega)$ have been applied. Because $H_{\alpha}(\delta)$ diverges as $\delta \rightarrow 0$ for $1<\alpha<3$, the small $\delta$ effectively sets the IR cutoff for the integral in (5.12). We see that for $1<\alpha<2$, the IR cutoff is at $x_{I R}^{2 \alpha} \simeq \delta^{4}$, giving $x_{I R} \simeq \delta^{2 / \alpha}$, and for $2<\alpha<3, x_{I R}^{4} \simeq \delta^{4}$, thus giving $x_{I R} \simeq \delta$. In the $\delta \rightarrow 0$ limit, the different infrared behaviors of the integrand in (5.12) between two ranges of $\alpha$ are due to the fact that the retarded Green's function in (3.7) in the small $\omega$ expansion is dominated by the mass term for $1<\alpha<2$ as a relevant operator in the IR limit, but the damping term for $2<\alpha<3$ where the mass term becomes an irrelevant operator. So, the integral with the divergent parts only can be estimated as

$$
H_{\alpha}(\delta \rightarrow 0) \propto\left\{\begin{array}{l}
\int_{\delta^{2 / \alpha}}^{1} d x \frac{x^{\alpha}}{x^{2 \alpha}} \propto \delta^{\frac{2}{\alpha}-2}, \quad 1<\alpha<2 ; \\
\int_{\delta}^{1} d x \frac{x^{\alpha}}{x^{4}} \propto \delta^{\alpha-3}, \quad 2<\alpha<3
\end{array}\right.
$$

The different behaviors of the position uncertainty in the different ranges of $\alpha$ lead to distinct saturated values of the entanglement entropy as we will see in the following.

At the late-time $\left(t \gg t_{n}\left(t \gg t_{b}\right)\right.$ for narrow (broad) resonance), the time dependence of the position uncertainty is mainly determined by the cut contributions to $G_{2}(t)$. Using (4.33) and the small $\omega$ approximation for $G_{2}(\omega)$ and $G_{H}(\omega)$, we obtain the asymptotic power law behavior as

$$
\begin{aligned}
\left\langle\hat{q}^{2}(t)\right\rangle_{F, \text { asy }} & \simeq-\frac{4}{M^{2}} \int_{0}^{\infty} \frac{d \omega}{2 \pi} G_{H}(\omega) \operatorname{Re}\left[G_{2}(\omega) \int_{t}^{\infty} G_{2}(\tau) e^{i \omega \tau}\right] \\
& \approx-\frac{2 \sin (\alpha \pi) \Gamma^{2}(1+\alpha) \sin ^{2}\left(\frac{\pi \alpha}{2}\right)}{M \Omega \pi(1+2 \alpha)} \Delta^{2}(\Omega t)^{-2 \alpha-1} .
\end{aligned}
$$

In figures 4 and 5 , we numerically check the asymptotic behavior of $\left\langle\hat{q}^{2}(t)\right\rangle_{F, \text { asy }}$ for the narrow and broad resonance cases respectively. This is done by using the small $\omega$ approximate of $G_{H}$ in (3.11) and $G_{2}(\omega)$ in (5.6). The numerics shows that the initial oscillation damps out and afterwards the position uncertainty settles to the power law relaxation at a rate $t^{-2 \alpha-1}$ as we find in (5.15). As compared with $\left\langle q^{2}(t)\right\rangle_{I}$ in $(5.4),\left\langle q^{2}(t)\right\rangle_{F, \text { asy }}$ dominates at late times, and to sum up, we have

$$
\left\langle q^{2}(t)\right\rangle=\left\langle q^{2}(\infty)\right\rangle_{F}-\frac{2 \sin (\alpha \pi) \Gamma^{2}(1+\alpha) \sin ^{2}\left(\frac{\pi \alpha}{2}\right)}{M \Omega \pi(1+2 \alpha)} \Delta^{2}(\Omega t)^{-2 \alpha-1}+\mathcal{O}\left((\Omega t)^{-2 \alpha-2}\right) .
$$

Using the operator relation $\hat{p}(t)=M \frac{d}{d t} \hat{q}(t)$, the cross correlation between the position and the momentum can be found by simply taking the time derivative of $\left\langle\hat{q}^{2}(t)\right\rangle$, and at late times, is given by

$$
\langle q(t) p(t)+p(t) q(t)\rangle \simeq \frac{2}{\pi} \sin (\alpha \pi) \Gamma^{2}(1+\alpha) \sin ^{2}\left(\frac{\pi \alpha}{2}\right) \Delta^{2}(\Omega t)^{-2 \alpha-2}+\mathcal{O}\left((\Omega t)^{-2 \alpha-3}\right)
$$




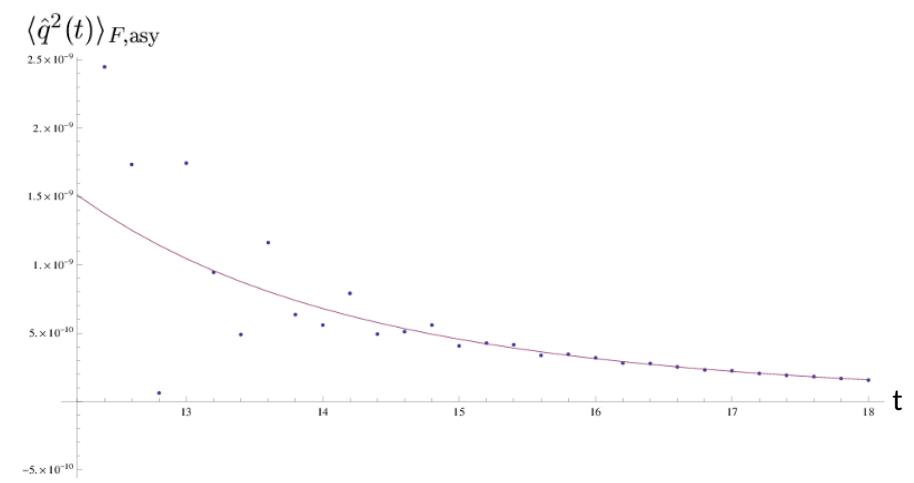

Figure 4. The dots show the numerical calculation of $\left\langle\hat{q}^{2}(t)\right\rangle_{F, \text { asy }}$, using the small $\omega$ approximate of $G_{H}$ in (3.11) and the $G_{2}(\omega)$ in (5.6). The solid line shows the power law time dependence as we find in eq. (5.15). In the plots, $\left\langle\hat{q}^{2}(t)\right\rangle_{F \text {, asy }}$ is in units of $\frac{2 \sin (\alpha \pi) \Gamma(1+\alpha) \sin \left(\frac{\pi \alpha}{2}\right) \Delta^{2}}{M \pi \Omega^{2 \alpha}}$ with the parameters chosen as in figure 2 for narrow resonance.

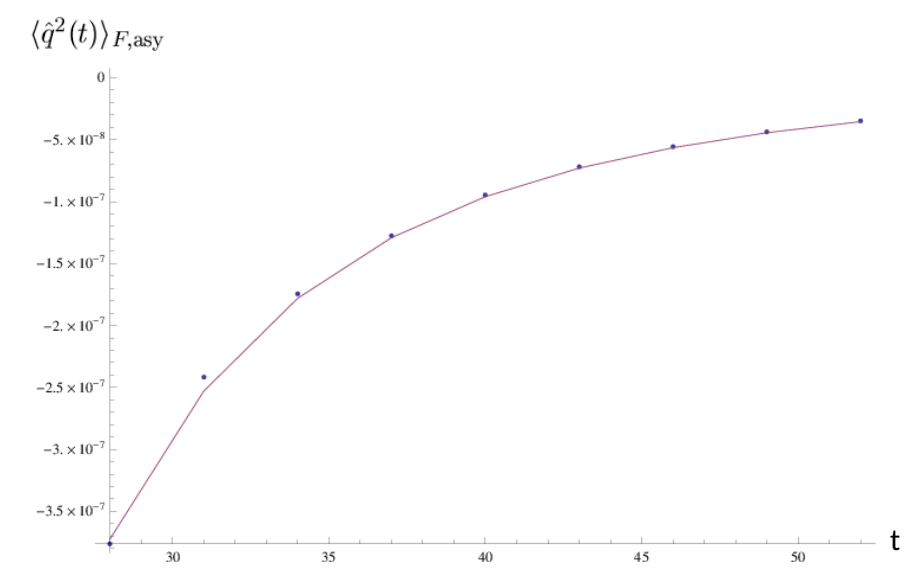

Figure 5. The same numerical calculation as in figure 4 with the parameters chosen for the broad resonance as in figure 3 .

with the vanishing saturated value. Following the same derivation, the late-time behavior of the momentum uncertainty is obtained as

$$
\begin{aligned}
&\left\langle\hat{p}^{2}(t)\right\rangle= m^{2} \dot{G}_{1}^{2}(t) G_{1}(t)\left\langle\hat{q}^{2}(0)\right\rangle+\dot{G}_{2}^{2}(t)\left\langle\hat{p}^{2}(0)\right\rangle+M \dot{G}_{1}(t) \dot{G}_{2}(t)\langle\hat{q}(0) \hat{p}(0)+\hat{p}(0) \hat{q}(0)\rangle \\
& \quad+\frac{1}{2} \int_{0}^{t} d \tau \int_{0}^{t} d \tau^{\prime} \dot{G}_{2}(t-\tau) \dot{G}_{2}\left(t-\tau^{\prime}\right)\left\langle\left\{\hat{\eta}(\tau), \hat{\eta}\left(\tau^{\prime}\right)\right\}\right\rangle \\
& \simeq\left\langle p^{2}(\infty)\right\rangle_{F}-\frac{2 \Omega M \sin (\alpha \pi)}{\pi(3+2 \alpha)} \Gamma(1+\alpha) \Gamma(3+\alpha) \sin ^{2}\left(\frac{\pi \alpha}{2}\right) \Delta^{2}(\Omega t)^{-2 \alpha-3}+\mathcal{O}\left((\Omega t)^{-2 \alpha-4}\right) .
\end{aligned}
$$

where $\left\langle\hat{p}^{2}(\infty)\right\rangle_{F}$ turns out to be finite as $\delta \rightarrow 0$ for all $\alpha>1$ and is given by

$$
\left\langle\hat{p}^{2}(\infty)\right\rangle_{F, \delta=0}=\frac{M^{2} z^{2} r_{b}^{z(3-\alpha)}}{\pi T_{n+1} S_{n}} \int_{0}^{1} d x \frac{x^{2} g(x)}{\left|B x^{2}+x f(x)\right|^{2}}
$$


Substituting (5.16), (5.17), and (5.18) into the definition of the $w(t)$ function (5.2), we then obtain the Von-Neumann entanglement entropy (5.1). We can similarly express the entanglement entropy as the saturated term and the term vanishes as $t \rightarrow \infty$

$$
S(t)=S(\infty)+S_{\mathrm{asy}}(t)
$$

To the leading order in the small- $\delta$ limit, the saturated value of the entanglement entropy can be found from $\left\langle\hat{q}^{2}(\infty)\right\rangle_{F}$ in (5.9), (5.11) and $\left\langle\hat{p}^{2}(\infty)\right\rangle_{F}$ in (5.19). We find that the saturated entanglement entropy has three qualitatively different behaviors for the following different ranges of $\alpha$,

$$
S(\infty) \simeq\left\{\begin{array}{l}
\left(1-\frac{1}{\alpha}\right) \ln \left(\frac{r_{b}^{z}}{\Omega}\right), \quad 1<\alpha<2 ; \\
\left(\frac{3}{2}-\frac{\alpha}{2}\right) \ln \left(\frac{r_{b}^{z}}{\Omega}\right), \quad 2<\alpha<3 ; \\
\left(\sqrt{W(\alpha)}+\frac{1}{2}\right) \ln \left(\sqrt{W(\alpha)}+\frac{1}{2}\right)-\left(\sqrt{W(\alpha)}-\frac{1}{2}\right) \ln \left(\sqrt{W(\alpha)}-\frac{1}{2}\right), \quad \alpha>3
\end{array}\right.
$$

where

$$
W(\alpha)=\frac{z \rho_{n}^{2} r_{b}^{2 z(2-\alpha)}}{\pi^{2} T_{n+1}^{2}} \int_{0}^{1} d x \frac{g(x)}{\left|B x^{2}+x f(x)\right|^{2}} \int_{0}^{1} d x \frac{x^{2} g(x)}{\left|B x^{2}+x f(x)\right|^{2}} .
$$

Here $W(\alpha)$ is the function $w(t \rightarrow \infty)$ in the case $\alpha>3$. For $\alpha>3$, the saturated value is finite and independent of the UV and IR cutoffs. In the unit that $\rho_{n} \simeq L^{-n-1}$, we find $W(\alpha) \simeq\left(\frac{l_{p}}{L}\right)^{2 z(\alpha-1)}\left(\frac{L}{r_{b}}\right)^{2 z(\alpha-2)}$, which is naturally very small. $W(\alpha)$ could be of the order one and consistent with the minimum uncertainty relation as $L \sim r_{b} \sim l_{p}$, but this leads to the breakdown of the classical gravity limit. Quantum gravity effect seems to become important for $\alpha>3$ and this deserves further study. Here we mainly focus on $1<\alpha<3$ but $\alpha \neq 2$ with the same range of $\alpha$ being considered in [27] in the case of the particle $(n=0)$. The saturated value of the Von Newmann entropy can be considered as a measurement of number of the degrees of freedom available to the system from the environment. Thus, for $1<\alpha<2$, our result indicates that the number of the effective degrees of freedom increases with increasing $\alpha$, while for $2<\alpha<3$, its number decreases with increasing $\alpha$. The maximum saturated entropy occurs as $\alpha$ approaches 2 , namely $z=n+2$, which happens as the mass term changes from an irrelevant operator for $\alpha<2$ to a relevant operator for $\alpha>2$. This is one of the main results in this paper.

The asymptotic behavior of the entanglement entropy toward the saturation is determined by $\left\langle\hat{q}^{2}(t)\right\rangle_{F, \text { asy }}$, which decays most slowly comparing to other terms in $w(t)$ at the late time with the behavior as

$$
S_{\text {asy }}(t) \simeq \frac{\left\langle\hat{q}^{2}(t)\right\rangle_{F, \text { asy }}}{\left\langle\hat{q}^{2}(\infty)\right\rangle_{F}}=-\frac{2 \sin (\alpha \pi) \Gamma^{2}(1+\alpha) \sin ^{2}\left(\frac{\pi \alpha}{2}\right)}{m \Omega \pi(1+2 \alpha)\left\langle\hat{q}^{2}(\infty)\right\rangle_{F}} \Delta^{2}(\Omega t)^{-2 \alpha-1} .
$$

The entanglement entropy shows the power-law relaxation at the late time. Moreover, the larger value of $z$ and smaller value of $n$ lead to smaller value of $\alpha$ and the smaller 
saturation rate. This is consistent with the field theory picture, where the relaxation is due to the energy flow from UV to IR degrees of freedom. The quantum critical theory in $d-1$ spacial dimension with the dispersion relation $E \propto k^{z}$ for the effective excitations has density of the states $\rho(E) \propto E^{-1+(d-1) / z}$ [27]. Since we consider the probed $n$-dimensional mirror, the effective density of the states probed by the mirror would be $\rho_{e}(E) \propto E^{-1+n / z}$. Thus the number of the modes decreases with the increase in $z$ or the decrease in $n$, which leads to the slower relaxation rate. This is a remarkable result. The proposed holographic model gives a very natural explanation to the relaxation rate that certainly deserves an experimental test.

\section{Comparison with the environment of relativistic free field theory}

The environment-induced effects on the system, for example, a point charge coupled to quantized electromagnetic fields [55] and a 2-dimensional moving mirror in the environmental quantum free fields [44] are to be summarized in this section in the paradigm of quantum Brownian motion $[4,5,49]$. In the linear response approximation, the equation of motion of either point charge or moving mirror can be cast into the Langevin equation in the form (4.9). Then, the self-energy in general can be effectively expressed in terms of the spectral density, $J(\omega)$ of the bath field denoted by the $\phi$ field as

$$
\Sigma_{\phi}(s)=-\frac{2}{\pi} \int_{0}^{\infty} d \omega J(\omega) \frac{\omega}{s^{2}+\omega^{2}},
$$

where $J(\omega) \propto \omega^{\beta}$. For example, a charged point particle coupled to the quantized electromagnetic field gives $\beta=3$ and a 2 -dimensional mirror moving in the medium of a relativistic free scalar field corresponds to $\beta=5$. The solution of the Langevin equation can similarly be constructed from the fundamental solutions $G_{\phi, 1}(t)$ and $G_{\phi, 2}(t)$, obeying the homogeneous part of (4.9) with the initial conditions (4.11) and (4.12). The Laplace transforms of the solutions are the same as the ones given by (4.14), with the self energy replaced by $\Sigma_{\phi}(s)$. The inverse Laplace transforms depend on the analytical structure of $\Sigma_{\phi}(s)$. It can be seen from (6.1) that the self-energy $\Sigma_{\phi}(s)$ can have a branch-cut along the imaginary $s$ axis. The real part and the imaginary part of $\Sigma_{\phi}(s)$ can be constructed by letting $s=i \omega \pm \epsilon$ in (6.1) where the resulting integral in the small $\epsilon$ limit is given by the principal value. Then we have,

$$
\Sigma_{\phi}(s=i \omega \pm \epsilon)=\operatorname{Re} \Sigma_{\phi}(\omega) \pm i \operatorname{Im} \Sigma_{\phi}(\omega)
$$

for $\omega>0$ where

$$
\begin{aligned}
& \operatorname{Re} \Sigma_{\phi}(\omega)=\frac{2}{\pi} \int_{\omega_{\mathrm{th}}}^{\Lambda} d \omega^{\prime} \frac{J\left(\omega^{\prime}\right) \omega^{\prime}}{\omega^{2}-\omega^{\prime 2}}, \\
& \operatorname{Im} \Sigma_{\phi}(\omega)=\frac{2}{\pi} \operatorname{sgn}(\omega) \int_{\omega_{\mathrm{th}}}^{\Lambda} d \omega^{\prime} J\left(\omega^{\prime}\right) \omega^{\prime} \delta\left(\omega^{2}-\omega^{\prime 2}\right) .
\end{aligned}
$$

Here we impose a threshold energy $\omega_{\text {th }}$ and a UV-cutoff $\Lambda$ in the definition of the selfenergy. The branch cut then splits into two segments $\left(i \omega_{\mathrm{th}}, i \Lambda\right)$, and also $\left(-i \Lambda,-i \omega_{\mathrm{th}}\right)$ (see figure 6). 


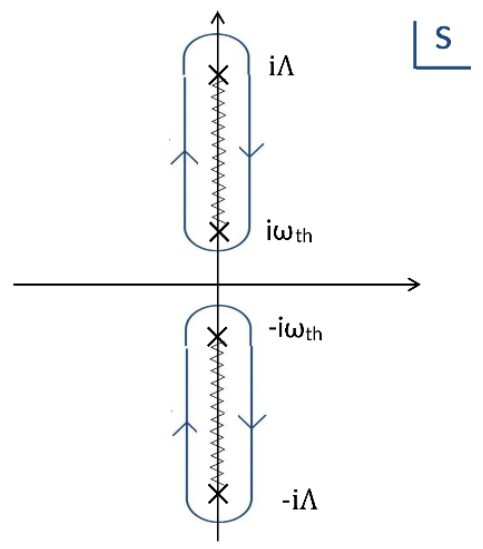

Figure 6. The contour for the inverse Laplace transform used to compute $G_{\phi, 1}(t)$ and $G_{\phi, 2}(t)$ in a bath of the relativistic free field. There exist the cuts but the poles are on other Riemann sheets.

As the Brownian particle is trapped in a harmonic potential with the frequency $\Omega$, the location of the poles in the inverse Laplace transform can be found by solving the pole equation similar to the one in (4.21). Following [49], it is convenient to define the renormalized self-energy and frequency,

$$
\Sigma_{\phi, R}(s)=\Sigma_{\phi}(s)-\Sigma_{\phi}(0) ; \quad \Omega_{R}^{2}=\Omega^{2}+\Sigma_{\phi}(0) .
$$

The subscript $R$ will be omitted in the following discussion for simplifying the notations. In the case that the interaction between the system and environment are weak and the environmental fields are free such as in $[44,55]$, the positions of the poles can be determined perturbatively with the corrections from environmental fields. As $\Omega<\omega_{\text {th }}$, the locations of the poles, $s_{p}$ is on the imaginary axis, leading to the late time oscillatory behavior. As $\omega_{\text {th }}<\Omega<\Lambda$, the free particle pole is on the branch-cut, and as can be seen from (6.4), the perturbative effects from the environment shift the free particle poles to the second Riemann sheet, then becoming the resonances [49]. This gives a clear mechanism that the decay of the system (a resonance) is due to the existence of the open channel that allows the energy transfer to the environment from the system. Thus, as $\omega_{\text {th }}<\Omega<\Lambda$, the inverse Laplace transform can be carried out by evaluating on the branch cuts, which gives

$$
G_{\phi, 2}(t)=\int_{0}^{\Lambda} d \omega \frac{4 \operatorname{Im} \Sigma_{\phi}(\omega)}{\left[\omega^{2}+\Omega^{2}+\operatorname{Re} \Sigma_{\phi}(\omega)\right]^{2}+\left[\operatorname{Im} \Sigma_{\phi}(\omega)\right]^{2}} \sin (\omega t) .
$$

This integral over the $\omega$ in the limit of the weak coupling between the system and bath exhibits a Breit-Wigner feature of the narrow resonance. The width of the resonance is determined by the imaginary part of the self-energy and its peak is located at the shifted resonance frequency around $\Omega$. For the intermediate times $\left(1 / \Omega \ll t \sim 1 / \Gamma_{\phi}\right)$, the real-time of $G_{\phi, 2}(t)$ can be approximated by

$$
G_{\phi, 2}(t) \simeq Z_{\phi} \cos \left[\Omega_{\phi} t+\theta_{\phi}\right] e^{-\Gamma_{\phi} t},
$$

where

$$
\Omega_{\phi} \simeq \Omega+\frac{\operatorname{Re} \Sigma_{\phi}\left(\Omega_{\phi}\right)}{2 \Omega_{\phi}} \quad \Gamma_{\phi} \simeq \frac{Z_{\phi} \operatorname{Im} \Sigma_{\phi}\left(\Omega_{\phi}\right)}{2 \Omega_{\phi}}
$$


with $\Omega \gg \Gamma_{\phi}$ obtained perturbatively. Moreover, the phase shift $\theta_{\phi}$ and $Z_{\phi}$ are

$$
Z_{\phi} \simeq\left[1-\frac{\partial \operatorname{Re} \Sigma_{\phi}\left(\Omega_{\phi}\right)}{\partial \Omega_{\phi}^{2}}\right]^{-1}, \quad \theta_{\phi} \simeq Z_{\phi} \frac{\partial \operatorname{Im} \Sigma_{\phi}\left(\Omega_{\phi}\right)}{\partial \Omega_{\phi}^{2}}
$$

which are all perturbatively small. Finally, in the much later time when $t \gg 1 / \Gamma_{\phi}, G_{\phi, 2}(t)$ decays in a power law in time as $1 / t^{\beta+1}$ determined by the small $\omega$ behavior of the integrand in (6.6) where the imaginary part of the self-energy is in (6.4) with the spectral density $J(\omega) \propto \omega^{\beta}$. The fundamental function $G_{\phi, 1}(t)$ shows the similar relaxation behavior. So, in the end, the entanglement entropy constructed from these fundamental functions $G_{\phi 1}$ and $G_{\phi, 2}$ will also exhibits the power law relaxation toward the saturation.

As a comparison, the self-energy of the system with the effects from the strongly coupled quantum critical fields shows the damping term of the form, $\omega^{\alpha}$ for non-integer $\alpha$ whereas for a relativistic field theories the powers of $\omega$ in the damping term is an integer as expected. Although the positions of the cuts in a complex $s$ plane are very different in both cases, at late times the system relaxes in a power law with the inverse of the powers of time $t$ due to the cut contributions. In particular, the existence of the cuts on the imaginary part of the self-energy for free relativistic fields can be explained by the energy transfer from the system to the bath via creating quantum excitations of the environment. However for quantum critical fields the cuts arise from the damping term with peculiar non-integer powers of $\omega$ dependence, giving the relaxation rate that also can be realized as the energy flow to the environment by counting the number of available modes of environmental quantum critical fields.

Moreover, the holographic approach allows us to include the non-perturbative effects from the environment. Thus, we find the complex-valued pole solutions for either broad or narrow resonances. However, the effects from relativistic free fields for a weak coupling between the system and the environment are treated perturbatively, giving a reliable result only on the narrow resonance with relatively small corrections from the environment. They all lead to the exponential decay in the intermediate time scales. To summarize, in both cases, the effects of the environment fields on the system share the same feature that the system decays exponentially from the initial state during the intermediate times given by the width of the resonance, and turns to the power law relaxation determined by the small $\omega$ behavior of the self-energy.

\section{$7 \quad$ Summary and outlook}

The main goal of this work is to understand the time evolution of the entanglement entropy between the $d$-dimensional strongly coupled quantum critical field with a dynamical exponent $z$ at zero temperature and a $n$-dimensional mirror using the method of holography. The dual description is a $n+1$-dimensional probe brane moving in $d+1$-dimensional Lifshitz geometry. The dynamics of the mirror can be realized from the motion of the brane at the boundary of the bulk. The interaction between the system and the environment may result in the loss of the information of the system, which can be measured by the von Neumann entropy, $S=-\operatorname{Tr} \rho_{r} \ln \rho_{r}$, computed from the reduced density matrix $\rho_{r}$ of the system. 
In the linear response approximation, we construct the holographic influence functional by tracing out the environment's degrees of freedom. Then, the stochastic effective action with the noise term manifested from quantum fluctuations of the environment field is obtained, from which the associated Heisenberg equations of the mirror with effects from the environment are derived. We consider the environment at zero temperature, and prepare an initial density matrix of the mirror trapped by a harmonic potential in its ground state. Two sources of the averages need to be dealt with: one is the average over the intrinsic quantum uncertainty of the mirror and the other is the stochastic average induced by the quantum fluctuations of the environment. When turning on the interaction of the system and the environment, the entanglement entropy between them in the linear response approximation can be found straightforwardly from the position and the momentum uncertainties as well as the expectation values of position-momentum cross correlations of the system by solving the Heisenberg equations. The self-energy of the mirror due to the effect of the environment not only gives the corrections to the poles but also shows the existence of the cuts in terms of the Laplace transformed variable $s$. We find that for $1<\alpha<3$ but $\alpha \neq 2$, the entanglement entropy at the late times follows a power law relaxation at a rate $1 / t^{2 \alpha+1}$ to the saturation, due to the cut contributions. We also find that the saturated values of the entanglement entropy show two qualitatively different behaviors in the regions $1<\alpha<2$ and $2<\alpha<3$. Moreover its relaxation dynamics can be explained by counting the number of the modes of the environments from the field theory perspective. We then compare with the system in the bath of relativistic free fields. The relaxation dynamics of the entanglement entropy in that case follows the similar power-law relaxation at the late times, where the existence of the cut has a clear explanation from the transfer of energy from the system to the environment.

The immediate extension of our work is to study the dynamics of relaxation and thermalization of the mirror coupled to quantum critical fields at finite temperature. Another extension of the current work is to consider two quantum systems coupled to one strongly coupled quantum field. In particular, we may explore the development of their quantum entanglement through the interaction with the common environment field. On the one hand, the environmental effects will induce quantum decoherence and disentanglement. On the other hand, the environment as suitably prepared or attuned to, can also assist in maintaining or even generating entanglement. To do so, one needs to extend the current holographic setup to include two objects moving in the asymptotic Lifshitz background.

Additionally, it will be of interest to compare our results with the time dependent entanglement entropy obtained based upon Ryu-Takayanagi conjecture [35, 36]. In [37] and [38], the relaxation of the entanglement entropy between two geometric regions in strongly coupled fields with $z=1$ was studied by preparing a non-stationary initial state and applying a global quench respectively. Their results show generic power law relaxation in the late times, although the detailed relaxation rate may depend on the shape of the regions. In their studies, the entanglement entropy saturates at some finite times, also determined by the geometry of the regions. In our case, the entanglement entropy shows the similar power law relaxation, but it saturates asymptotically as the time goes to $t \rightarrow \infty$ instead. This may due to the fact that the perturbation in their cases is a global quench, 
whereas in our case the perturbation is local so it takes infinite time to establish the entanglement to the whole environment. The environment fields in their case are for quantum critical field with $z=1$ ( $\alpha$ is integer-valued) and also in finite temperature. It will be interesting to extend our study to those cases and make the comparison. Moreover, in [56], they studied the global quench in the Lifshitz background that extended the work in [38]. They found that in the case that the entanglement boundary is a sphere, the late time saturation rate of the entanglement entropy is independent of $z$, but the early time power-law growth has the similar dependence on $\alpha=1+\frac{2}{z}$ as in our case with local perturbation. The detailed comparison deserves further study.

\section{Acknowledgments}

This work was supported in part by the Ministry of Science and Technology, Taiwan.

\section{Brief summary of the holographic influence functional method}

Consider the Lifshitz black hole background with the metric

$$
d s^{2}=-r^{2 z} f(r) d t^{2}+\frac{d r^{2}}{f(r) r^{2}}+r^{2} d x_{i} d x_{i}
$$

where $f(r) \rightarrow 1$ for $r \rightarrow \infty$ and $f(r) \simeq c\left(r-r_{h}\right)$ near the black brane horizon $r_{h}$ with $c=(d+z-1) / r_{h}$. With the same notations and assumptions as in the main text, the DBI action for the $n+1$-dimensional probe brane in the Lifshitz black hole for small perturbation $X^{I}$ around the stationary configuration is given by

$$
S_{\mathrm{DBI}}^{T} \approx \text { constant }-\frac{T_{n+1}}{2} \int d r d t d x_{1} d x_{2} \ldots d x_{n}\left(r^{z+n+3} f(r) X^{\prime I} X^{I I}-\frac{\dot{X}^{I} \dot{X}^{I}}{f(r) r^{z-n-1}}\right) .
$$

The equation of motion for $X^{I}$ 's in the Fourier space, $X_{\omega}^{I}(r) e^{-i \omega t}$ can then be derived as follows

$$
\frac{\partial}{\partial r}\left(r^{z+n+3} f(r) \frac{\partial}{\partial r} X_{\omega}^{I}(r)\right)+\frac{\omega^{2}}{r^{z-n-1} f(r)} X_{\omega}^{I}(r)=0 .
$$

The solution can be expressed in terms of two linearly independent solutions with the properties $\mathcal{X}_{\omega}(r) \underset{r \rightarrow r_{h}}{\propto} e^{+i \omega r_{*}}$ and $\mathcal{X}_{\omega}^{*}(r) \underset{r \rightarrow r_{h}}{\propto} e^{-i \omega r_{*}}$, where $r^{*}=\int d r f(r)^{-1} r^{-z-1}$, and the normalization condition $\mathcal{X}_{\omega}\left(r_{b}\right)=1$. Since the different components of $X_{\omega}^{I}$ are decoupled in the linearized equation of motion, we may just focus on one of the directions $X^{I}$ and denote it by $Q(t, r)$. As described in the main text we introduce $Q^{+}\left(t, r_{1}\right)$ and $Q^{-}\left(t, r_{2}\right)$, which correspond to the branes living in two outside regions in the maximally extended Lifshitz black hole geometry. Following [14], which is consistent with [46, 47], $Q^{ \pm}(\omega, r)$ are then uniquely determined with extra boundary conditions

$$
q^{ \pm}(t)=Q^{ \pm}\left(t, r_{b}\right)
$$


to be

$$
\begin{aligned}
& Q^{+}\left(\omega, r_{1}\right)=\frac{1}{1-e^{-\frac{\omega}{T}}}\left[\left(q^{-}(\omega)-e^{-\frac{\omega}{T}} q^{+}(\omega)\right) \mathcal{X}_{\omega}\left(r_{1}\right)+\left(q^{+}(\omega)-q^{-}(\omega)\right) \mathcal{X}_{\omega}^{*}\left(r_{1}\right)\right], \\
& Q^{-}\left(\omega, r_{2}\right)=\frac{1}{1-e^{-\frac{\omega}{T}}}\left[\left(q^{-}(\omega)-e^{-\frac{\omega}{T}} q^{+}(\omega)\right) \mathcal{X}_{\omega}\left(r_{1}\right)+e^{-\frac{\omega}{T}}\left(q^{+}(\omega)-q^{-}(\omega)\right) \mathcal{X}_{\omega}^{*}\left(r_{1}\right)\right],
\end{aligned}
$$

where $q^{ \pm}(\omega)$ is the Fourier transform of $q^{ \pm}(t)$, which will be identified with the mirror's position in the close-time-path formalism. This solution is then substituted into the classical action, we then obtain the holographic influence functional

$$
\begin{gathered}
F\left(q^{+}, q^{-}\right)=S_{\mathrm{DBI}}^{\mathrm{on}-\text { shell }}\left(Q^{+}\right)-S_{\mathrm{DBI}}^{\mathrm{on}-\mathrm{shell}}\left(Q^{-}\right) \\
=-T_{n+1} S_{n} r_{b}^{z+n+3} \int \frac{d \omega}{2 \pi}\left(Q^{+}\left(-\omega, r_{b}\right) \partial_{r} Q^{+}\left(\omega, r_{b}\right)-Q^{-}\left(-\omega, r_{b}\right) \partial_{r} Q^{-}\left(\omega, r_{b}\right)\right) \\
=-\int \frac{d \omega}{2 \pi}\left\{q^{+}(-\omega)\left[i \operatorname{Re} G_{R}(\omega)-\left(1+2 n_{\omega}\right) \operatorname{Im} G_{R}(\omega)\right] q^{-}(\omega)\right. \\
+q^{-}(-\omega)\left[-i \operatorname{Re} G_{R}(\omega)-\left(1+2 n_{\omega}\right) \operatorname{Im} G_{R}(\omega)\right] q^{-}(\omega) \\
-q^{+}(-\omega)\left[-2 n_{\omega} e^{\frac{\omega}{2 T}} \operatorname{Im} G_{R}(\omega)\right] q^{-}(\omega) \\
\left.-q^{-}(-\omega)\left[-2\left(1+n_{\omega}\right) e^{-\frac{\omega}{2 T}} \operatorname{Im} G_{R}(\omega)\right] q^{+}(\omega)\right\}
\end{gathered}
$$

where $S_{n}$ is mirror's volume and $G_{R}^{(T)}(\omega)=T_{n+1} S_{n} r_{b}^{z+n+3} \mathcal{X}_{-\omega}\left(r_{b}\right) \partial_{r} \mathcal{X}_{\omega}\left(r_{b}\right)$ is the retarded Green function at the finite temperature $T$, as can be seen from (2.6) and (2.8). In the zero temperature limit, there is an exact expression for the solution in (8.3), satisfying the desired boudnary conditions,

$$
\mathcal{X}_{\omega}(r)=\frac{r_{b}^{\frac{z+n+2}{2}}}{r^{\frac{z+n+2}{2}}} \frac{H_{\frac{n+2}{2}+\frac{1}{2}}^{(1)}\left(\frac{\omega}{z r^{z}}\right)}{H_{\frac{n+2}{2 z}+\frac{1}{2}}^{(1)}\left(\frac{\omega}{z r_{b}^{z}}\right)} .
$$

Hence the zero-temperature retarded Green's function for $\omega>0$ can be found to be,

$$
G_{R}(\omega)=-T_{n+1} S_{n} \omega r_{b}^{n+2} \frac{H_{\frac{n+2}{2 z}-\frac{1}{2}}^{(1)}\left(\frac{\omega}{z r_{b}^{z}}\right)}{H_{\frac{n+2}{2 z}+\frac{1}{2}}^{(1)}\left(\frac{\omega}{z r_{b}^{z}}\right)} .
$$

The zero-temperature Hadamard function for $\omega>0$ can also be found using (2.6) and (2.8),

$$
G_{H}(\omega)=\frac{2 z}{\pi} r_{b}^{n+2+z} \frac{T_{n+1} S_{n}}{J_{\frac{n+2}{2 z}+\frac{1}{2}}^{2}\left(\frac{\omega}{z r_{b}^{z}}\right)+Y_{\frac{n+2}{2 z}+\frac{1}{2}}^{2}\left(\frac{\omega}{z r_{b}^{z}}\right)} .
$$

Open Access. This article is distributed under the terms of the Creative Commons Attribution License (CC-BY 4.0), which permits any use, distribution and reproduction in any medium, provided the original author(s) and source are credited. 


\section{References}

[1] S. Sachdev, Quantum phase transitions, Cambridge University Press, Cambridge (1999).

[2] M.A. Nielsen and I.L. Chuang, Quantum Computation and Quantum Information, Cambridge University Press, Cambridge (2000).

[3] D. Harlow, Jerusalem Lectures on Black Holes and Quantum Information, Rev. Mod. Phys. 88 (2016) 015002 [arXiv: 1409.1231] [INSPIRE].

[4] E. Calzetta and B.-L. Hu Nonequilibrium Quantum Field Theory, Cambridge Monographs on Mathematical Physics, Cambridge University Press, Cambridge (2008).

[5] N.P. Breuer and F. Petruccione, The Theory of Open Quantum Systems, Oxford University Press, Oxford (2007).

[6] R.P. Feynman and F.L. Vernon Jr., The Theory of a general quantum system interacting with a linear dissipative system, Annals Phys. 24 (1963) 118 [Annals Phys. 281 (2000) 547] [INSPIRE].

[7] A.O. Caldeira and A.J. Leggett, Path integral approach to quantum Brownian motion, Physica A 121 (1983) 587.

[8] A.O. Caldeira and A.J. Leggett, Influence of dissipation on quantum tunneling in macroscopic systems, Phys. Rev. Lett. 46 (1981) 211 [INSPIRE].

[9] A.O. Caldeira and A.J. Leggett, Quantum tunneling in a dissipative system, Annals Phys. 149 (1983) 374 [INSPIRE]

[10] J.S. Schwinger, Brownian motion of a quantum oscillator, J. Math. Phys. 2 (1961) 407 [INSPIRE].

[11] L.V. Keldysh, Diagram technique for nonequilibrium processes, Zh. Eksp. Teor. Fiz. 47 (1964) 1515 [INSPIRE].

[12] H. Grabert, P. Schramm and G.L. Ingold, Quantum Brownian motion: The Functional inegral approach, Phys. Rept. 168 (1988) 115 [INSPIRE].

[13] D.T. Son and D. Teaney, Thermal Noise and Stochastic Strings in AdS/CFT, JHEP 07 (2009) 021 [arXiv:0901.2338] [INSPIRE].

[14] C.-P. Yeh, J.-T. Hsiang and D.-S. Lee, Holographic influence functional and its application to decoherence induced by quantum critical theories, Phys. Rev. D 91 (2015) 046009 [arXiv: 1410.7111] [INSPIRE].

[15] J.M. Maldacena, The Large $N$ limit of superconformal field theories and supergravity, Int. $J$. Theor. Phys. 38 (1999) 1113 [hep-th/9711200] [INSPIRE].

[16] S.S. Gubser, I.R. Klebanov and A.M. Polyakov, Gauge theory correlators from noncritical string theory, Phys. Lett. B 428 (1998) 105 [hep-th/9802109] [INSPIRE].

[17] E. Witten, Anti-de Sitter space and holography, Adv. Theor. Math. Phys. 2 (1998) 253 [hep-th/9802150] [INSPIRE].

[18] S.A. Hartnoll, Lectures on holographic methods for condensed matter physics, Class. Quant. Grav. 26 (2009) 224002 [arXiv:0903.3246] [INSPIRE].

[19] C.P. Herzog, A. Karch, P. Kovtun, C. Kozcaz and L.G. Yaffe, Energy loss of a heavy quark moving through $N=4$ supersymmetric Yang-Mills plasma, JHEP 07 (2006) 013 [hep-th/0605158] [INSPIRE]. 
[20] S.S. Gubser, Drag force in AdS/CFT, Phys. Rev. D 74 (2006) 126005 [hep-th/0605182] [INSPIRE].

[21] J. Casalderrey-Solana and D. Teaney, Heavy quark diffusion in strongly coupled $N=4$ Yang-Mills, Phys. Rev. D 74 (2006) 085012 [hep-ph/0605199] [INSPIRE].

[22] G.C. Giecold, E. Iancu and A.H. Mueller, Stochastic trailing string and Langevin dynamics from AdS/CFT, JHEP 07 (2009) 033 [arXiv:0903.1840] [INSPIRE].

[23] J. Casalderrey-Solana, K.-Y. Kim and D. Teaney, Stochastic String Motion Above and Below the World Sheet Horizon, JHEP 12 (2009) 066 [arXiv:0908.1470] [INSPIRE].

[24] S. Caron-Huot, P.M. Chesler and D. Teaney, Fluctuation, dissipation and thermalization in non-equilibrium AdS $S_{5}$ black hole geometries, Phys. Rev. D 84 (2011) 026012 [arXiv: 1102 .1073] [INSPIRE].

[25] J. Boer, V. Hubeny, M. Rangamani and M. Shigemori, Brownian motion in AdS/CFT, JHEP 07 (2009) 094 [arXiv: 0812.5112].

[26] V. Hubeny and M. Rangamani, A Holographic view on physics out of equilibrium, Adv. High Energy Phys. 2010 (2010) 297916 [INSPIRE].

[27] D. Tong and K. Wong, Fluctuation and Dissipation at a Quantum Critical Point, Phys. Rev. Lett. 110 (2013) 061602 [arXiv:1210.1580] [INSPIRE].

[28] S.A. Hartnoll, J. Polchinski, E. Silverstein and D. Tong, Towards strange metallic holography, JHEP 04 (2010) 120 [arXiv:0912.1061] [INSPIRE].

[29] C.-P. Yeh, J.-T. Hsiang and D.-S. Lee, Holographic Approach to Nonequilibrium Dynamics of Moving Mirrors Coupled to Quantum Critical Theories, Phys. Rev. D 89 (2014) 066007 [arXiv: 1310.8416] [INSPIRE].

[30] C.-P. Yeh and D.-S. Lee, Subvacuum effects in quantum critical theories from a holographic approach, Phys. Rev. D 93 (2016) 126006 [arXiv:1510.05778] [InSPIRE].

[31] D.-S. Lee and C.-P. Yeh, A holographic description of negative energy states, JHEP 09 (2016) 059 [arXiv : 1606. 02420] [InSPIRE].

[32] D.-S. Lee and C.-P. Yeh, Environment-induced uncertainties on moving mirrors in quantum critical theories via holography, Annals Phys. 394 (2018) 316 [arXiv:1706. 08283] [INSPIRE].

[33] D. Giataganas, D.-S. Lee and C.-P. Yeh, Quantum Fluctuation and Dissipation in Holographic Theories: A Unifying Study Scheme, JHEP 08 (2018) 110 [arXiv:1802.04983] [INSPIRE].

[34] D. Giataganas, Stochastic Motion of Heavy Quarks in Holography: A Theory-Independent Treatment, PoS (CORFU2017) 032 (2018) [arXiv: 1805.09011] [INSPIRE].

[35] S. Ryu and T. Takayanagi, Holographic derivation of entanglement entropy from AdS/CFT, Phys. Rev. Lett. 96 (2006) 181602 [hep-th/0603001] [INSPIRE].

[36] V.E. Hubeny, M. Rangamani and T. Takayanagi, A Covariant holographic entanglement entropy proposal, JHEP 07 (2007) 062 [arXiv:0705.0016] [INSPIRE].

[37] T. Hartman and J. Maldacena, Time Evolution of Entanglement Entropy from Black Hole Interiors, JHEP 05 (2013) 014 [arXiv:1303.1080] [INSPIRE].

[38] H. Liu and S.J. Suh, Entanglement Tsunami: Universal Scaling in Holographic Thermalization, Phys. Rev. Lett. 112 (2014) 011601 [arXiv:1305.7244] [INSPIRE]. 
[39] S. Kachru, X. Liu and M. Mulligan, Gravity duals of Lifshitz-like fixed points, Phys. Rev. D 78 (2008) 106005 [arXiv:0808.1725] [InSPIRE].

[40] U. Gürsoy, E. Plauschinn, H. Stoof and S. Vandoren, Holography and ARPES Sum-Rules, JHEP 05 (2012) 018 [arXiv:1112.5074] [INSPIRE].

[41] M. Alishahiha, M.R. Mohammadi Mozaffar and A. Mollabashi, Fermions on Lifshitz Background, Phys. Rev. D 86 (2012) 026002 [arXiv:1201.1764] [INSPIRE].

[42] U. Gürsoy, V. Jacobs, E. Plauschinn, H. Stoof and S. Vandoren, Holographic models for undoped Weyl semimetals, JHEP 04 (2013) 127 [arXiv: 1209.2593] [INSPIRE].

[43] D. Rokhsar and S. Kivelson, Superconductivity and the Quantum Hard-Core Dimer Gas, Phys. Rev. Lett. 61 (1988) 2376.

[44] C.-H. Wu and D.-S. Lee, Nonequilibrium dynamics of moving mirrors in quantum fields: Influence functional and Langevin equation, Phys. Rev. D 71 (2005) 125005 [quant-ph/0501127] [INSPIRE].

[45] M. Taylor, Non-relativistic holography, arXiv:0812.0530 [INSPIRE].

[46] C.P. Herzog and D.T. Son, Schwinger-Keldysh propagators from AdS/CFT correspondence, JHEP 03 (2003) 046 [hep-th/0212072] [INSPIRE].

[47] D.T. Son and A.O. Starinets, Minkowski space correlators in AdS/CFT correspondence: Recipe and applications, JHEP 09 (2002) 042 [hep-th/0205051] [INSPIRE].

[48] E. Joos and H.D. Zeh, The Emergence of classical properties through interaction with the environment, Z. Phys. B 59 (1985) 223 [INSPIRE].

[49] S.M. Alamoudi, D. Boyanovsky, H.J. de Vega and R. Holman, Quantum kinetics and thermalization in an exactly solvable model, Phys. Rev. D 59 (1999) 025003 [hep-ph/9806235] [INSPIRE].

[50] D. Nagy and P. Domokos, Nonequilibrium Quantum Criticality and Non-Markovian Environment: Critical Exponent of a Quantum Phase Transition, Phys. Rev. Lett. 115 (2015) 043601.

[51] M. Al-Ali and T. Vojta, Quantum phase transition of the sub-Ohmic rotor model, Phys. Rev. B 84 (2011) 195136.

[52] J.D. Jackson, Classical Electrodynamics, 2nd edition, Wiley, New York (1975).

[53] G.T. Horowitz and R.M. Wald, Dynamics of Einstein's Equation Modified by a Higher Order Derivative Term, Phys. Rev. D 17 (1978) 414 [InSPIRE].

[54] L. Parker and J.Z. Simon, Einstein equation with quantum corrections reduced to second order, Phys. Rev. D 47 (1993) 1339 [gr-qc/9211002] [InSPIRE].

[55] J.-T. Hsiang and D.-S. Lee, Influence on electron coherence from quantum electromagnetic fields in the presence of conducting plates, Phys. Rev. D 73 (2006) 065022 [hep-th/0512059] [INSPIRE].

[56] P. Fonda, L. Franti, V. Keränen, E. Keski-Vakkuri, L. Thorlacius and E. Tonni, Holographic thermalization with Lifshitz scaling and hyperscaling violation, JHEP 08 (2014) 051 [arXiv: 1401.6088] [INSPIRE]. 